\title{
XMM-NEWTON MONITORING OF THE CLOSE PRE-MAIN-SEQUENCE BINARY AK SCO. EVIDENCE OF TIDE-DRIVEN FILLING OF THE INNER GAP IN THE CIRCUMBINARY DISK
}

\author{
Ana Inés Gómez de Castro ${ }^{1}$, Javier López-Santiago ${ }^{2}$, Antonio Talavera ${ }^{3}$, A. Yu. Sytov $^{4}$, and D. Bisikalo ${ }^{4}$ \\ ${ }^{1}$ S. D. Astronomía y Geodesia and Instituto de Matemática Interdisciplinar, Fac. de CC Matemáticas, Universidad Complutense, E-28040 Madrid, Spain \\ ${ }^{2}$ Departamento de Astrofísica, Fac de CC Físicas, Universidad Complutense, E-28040 Madrid, Spain \\ ${ }^{3}$ European Space Astronomy Center, Villanueva de la Cañada, E-28691, Madrid, Spain \\ ${ }^{4}$ Institute of Astronomy of the Russian Academy of Sciences, Pyatnitskaya St. 48, 109017 Moscow, Russia \\ Received 2012 May 31; accepted 2013 January 29; published 2013 March 7
}

\begin{abstract}
AK Sco stands out among pre-main-sequence binaries because of its prominent ultraviolet excess, the high eccentricity of its orbit, and the strong tides driven by it. AK Sco consists of two F5-type stars that get as close as $11 R_{*}$ at periastron passage. The presence of a dense $\left(n_{e} \sim 10^{11} \mathrm{~cm}^{-3}\right)$ extended envelope has been unveiled recently. In this article, we report the results from an XMM-Newton-based monitoring of the system. We show that at periastron, X-ray and UV fluxes are enhanced by a factor of $\sim 3$ with respect to the apastron values. The X-ray radiation is produced in an optically thin plasma with $T \sim 6.4 \times 10^{6} \mathrm{~K}$ and it is found that the $N_{\mathrm{H}}$ column density rises from $0.35 \times 10^{21} \mathrm{~cm}^{-2}$ at periastron to $1.11 \times 10^{21} \mathrm{~cm}^{-2}$ at apastron, in good agreement with previous polarimetric observations. The UV emission detected in the Optical Monitor band seems to be caused by the reprocessing of the high-energy magnetospheric radiation on the circumstellar material. Further evidence of the strong magnetospheric disturbances is provided by the detection of line broadening of $278.7 \mathrm{~km} \mathrm{~s}^{-1}$ in the N v line with Hubble Space Telescope/Space Telescope Imaging Spectrograph. Numerical simulations of the mass flow from the circumbinary disk to the components have been carried out. They provide a consistent scenario with which to interpret AK Sco observations. We show that the eccentric orbit acts like a gravitational piston. At apastron, matter is dragged efficiently from the inner disk border, filling the inner gap and producing accretion streams that end as ring-like structures around each component of the system. At periastron, the ring-like structures come into contact, leading to angular momentum loss, and thus producing an accretion outburst.
\end{abstract}

Key words: binaries: spectroscopic - magnetic fields - stars: pre-main sequence

Online-only material: color figures

\section{INTRODUCTION}

Late-type, pre-main-sequence (PMS) stars, T Tauri Stars (TTSs), are characterized by their strong radiation excess at high energies, from UV to X-ray wavelengths. There has been much discussion regarding the source of this excess. In the 1980s and early 1990s it was thought that this excess was associated with an enhancement of the magnetic activity caused by the deep convective layers of the TTSs prior to stellar stabilization on the main sequence (Giampapa et al. 1981; Calvet et al. 1985). Later on, the realization that accretion itself could be the source of energy drove the development of several models where both UV and X-ray excesses were produced by accretion shocks, material free-falling along the stellar field lines onto a shock front at the stellar surface (Lamzin 1998; Gómez de Castro \& Lamzin 1999; Gullbring et al. 2000; Günther et al. 2007). The shock front temperature may reach a few $\times 10^{6} \mathrm{~K}$, hence producing soft X-ray emission and UV excess radiation. In 2001, it was realized that outflows from these systems are hot enough to contribute to the UV excess (Gómez de Castro \& Verdugo 2001); the magnetically mediated interaction between the Keplerian accretion disk and the star basically transforms angular momentum from the disk storage into mechanical energy (outflows) and heating. The phenomenon is non-stationary and it is controlled by the rotation period and the diffusion timescale. There is general consensus on the co-existence of two components to this outflow: a warm high-density component associated with a centrifugally driven wind from the disk, and a hot low-density component associated with plasmoid ejection from the magnetic interface between the stellar field and the disk (von Rekowski \& Brandenburg 2004; Gómez de Castro \& von Rekowski 2011). Thus, jets are expected to have a non-stationary, magnetized, hot component. Indeed, $\mathrm{X}$-ray emission has been detected from some protostellar jets (Favata et al. 2002; Bonito et al. 2007; Guedel et al. 2008).

Current understanding of the physics of star formation indicates that magnetic activity, accretion, and outflow are contributing to the high-energy excess of the PMS systems. The stellar magnetosphere acts as the interface between the various dynamical components (disk, star) and channels/dials the flows driven by them (accretion and winds/jets).

The magnetospheres of PMS stars are very powerful and extend 2-8 stellar radii to the limits of the inner disk boundary (Johns-Krull 2007; Gómez de Castro \& Marcos-Arenal 2012, hereafter GdCMA2012). They are powered by the stellar magnetic field, the accreting infalling material, and shearing at the boundary between the magnetosphere and the inner disk (see Hartmann 2009 for a recent review and Gómez de Castro 2009a for the UV radiative output of this engine). Magnetospheric heating processes during PMS evolution are poorly studied, as well as the relative relevance of the contributions mentioned above. Close PMS binaries provide an ideal laboratory to study magnetospheric physics since the coupling between the stellar magnetospheres and the disk is broken and gravity controls the mass transfer from the inner border of the disk to the stars. $\mathrm{Nu}$ merical simulations predict the dynamical clearing of the inner region of the circumbinary disk (Artymowicz \& Lubow 1994; Bate \& Bonnel 1997; Kaigorodov et al. 2010; Fateeva et al. 2011; de Val-Borro et al. 2011). Indeed, recent infrared studies have shown that most of the (few) known PMS spectroscopic 
Table 1

AK Sco Main Parameters

\begin{tabular}{lcc}
\hline \hline Property & Value & Source \\
\hline Projected semimajor axis & $a \sin i=30.77 \pm 0.12 R_{\odot}$ & A1989 \\
Eccentricity & $e=0.47$ & A1989 and A2003 \\
Orbital period & $P=13.609 \mathrm{~d}$ & A1989 and A2003 \\
Inclination & $i=65^{\circ}-70^{\circ}$ & A2003 \\
Age & $10-30 \mathrm{Myr}$ & A2003 \\
Spectral type & F5 & A2003 \\
Stellar mass & $M_{*}=1.35 \pm 0.07 M_{\odot}$ & A2003 \\
Radius & $R_{*}=(1.59 \pm 0.35) R_{\odot}$ & A2003 \\
Projected rotation velocity & $v \sin i=18.5 \pm 1.0 \mathrm{~km} \mathrm{~s}^{-1}$ & A2003 \\
Bolometric flux & $6.33 \times 10^{-9} \mathrm{erg} \mathrm{s} \mathrm{cm}^{-2}$ & A1989 \\
Extinction: $A_{V}$ & $0.5 \mathrm{mag}$ & MBB2005 \\
Extinction: $R$ & 4.3 & MBB2005 \\
Distance & $102.8 \mathrm{pc}$ & van Leeuwen (2007) \\
\hline
\end{tabular}

binaries (PMS-SBs; Mathieu et al. 1997 and references therein) do not display significant infrared excess in the $1-5 \mu \mathrm{m}$ range suggesting cleared regions within the circumbinary disk (Jensen \& Mathieu 1997). The size of the gaps is consistent with the theoretical predictions for dynamically clearing. However, PMSSBs display UV excesses as high as their single counterparts do (enhanced emission of the so-called chromospheric/transition region tracers). This suggests that the disk-star locking is not the dominant source of magnetospheric heating; rather it seems to be controlled by the stellar activity and the accretion flow.

AK Sco stands out among the PMS-SBs because of the evidence of transient material filling the gap and its prominent ultraviolet excess. The high eccentricity of AK Sco's orbit triggers strong tides in the inner border of the circumbinary disk as well as in both components; AK Sco is made of two F5-type stars that get as close as $11 R_{*}$ at periastron passage (see Table 1 for a summary of AK Sco properties). Moreover, recent UV observations have unveiled the presence of dense and extended magnetospheres around each component (Gómez de Castro 2009b). Each stellar magnetosphere extends to $\sim 1.7 R_{*}$ and is highly turbulent; the region is disturbed by a velocity field $\left(\sigma \simeq 100 \mathrm{~km} \mathrm{~s}^{-1}\right.$ ) that significantly exceeds the sound speed in the UV radiating plasma. The radiative loses of the magnetospheres $\left(0.04 L_{\odot}\right.$ per star) cannot be accounted solely by the dissipation of energy from the tidal wave; hence, the contribution of the accretion flow needs to be taken into account. However, no signs of enhanced accretion at periastron passage have been reported from the optical $\mathrm{H} \alpha / \mathrm{H} \beta$ profiles (Andersen et al. 1989, hereafter A1989; Alencar et al. 2003, hereafter A2003). This is not the generic case for PMS-SBs; for instance, $\mathrm{KH} 15 \mathrm{D}$ shows phase-dependent variations of its $\mathrm{H} \alpha$ profiles, which have been interpreted as a sign of an accretion stream member (Hamilton et al. 2012).

In this article, we present evidence of the enhancement of energy release at periastron based on the X-ray/UV monitoring of AK Sco with the XMM-Newton space telescope. The presence of extended structures around each component of the binary system is confirmed by recent observations of AK Sco obtained from the Hubble Space Telescope (HST) archive. Also evidence of an enhanced dragging of material from the inner disk border into the gap at apastron is reported. Numerical simulations of the stars-disk interaction have been carried out to analyze the observations. It is shown that the high eccentricity of AK Sco orbit acts like a gravitational piston that efficiently drags matter from the disk border at apastron, feeding an enhanced mass infall at periastron. In Section 2, we describe the XMM-Newton observations and data reduction as well as the archival data obtained from the International Ultraviolet Explorer (IUE) and the HST. In Section 3, we present the analysis of these data and the obtained results. The binary-disk model and the numerical setup developed to study the evolution of the system is described in Section 4. In Section 5, we propose the general framework to interpret the observations. Finally, a brief summary is provided in Section 6.

\section{OBSERVATIONS AND DATA REDUCTION}

\subsection{XMM-Newton Observations and Data Reduction}

The XMM-Newton monitoring of AK Sco was carried out from 2011 March 15 to 2011 March 22. The observations were performed at phases ${ }^{5} 0.99,0.15$, and 0.48 , corresponding to observation identifications, ID. 0651870201, ID. 0651870301, and ID. 0651870401, respectively. The observing log is presented in Table 2.

The data obtained in the three XMM-Newton observations have been reduced using the standard Science Analysis System (SAS) developed by the XMM-Newton project. SAS allows the user to apply all instrumental corrections and calibrations corresponding to the instruments on board XMM-Newton. SAS version 11 has been used for the reduction of both EPIC and OM data.

\subsubsection{EPIC Data}

The European Photon Imaging Cameras (EPIC) were used in each observation in full-frame mode with the thick filter for an exposure time of approximately $25 \mathrm{ks}$. The standard procedure was followed for EPIC data reduction and calibration. First, calibrated file events were created using SAS tasks epproc and emproc for each observation independently.

Light curves obtained from events detected in the whole field of view with energy $E>10 \mathrm{keV}$ were explored to identify high background periods that did not appear in any of the three observations. The event lists were then cleaned for bad events and noise. The resultant event files were used for the extraction of source light curves and spectra of AK Sco.

\subsubsection{Optical and UV Monitor Observations and Data Reduction}

The Optical and UV Monitor (OM) was operated in User Defined Mode, with the $U V M 2$ filter (effective wavelength $\sim 2310 \AA$ ). Four exposures of 4400 s were obtained in each observation. Two detector windows were defined in each exposure: an image-mode window of $5 \times 5 \mathrm{arcmin}^{2}$ and a fast-mode one of $12 \times 12 \operatorname{arcsec}^{2}$ centered on AK Sco. The fast-mode window allowed us to obtain data with a time resolution of $1 \mathrm{~s}$.

Aperture photometry has been performed on the OM images, obtained with the $U V M 2$ filter. The results obtained with SAS, corrected count rates and the corresponding AB magnitudes, are given in Table 3.

Detailed light curves have been derived from the fast-mode OM event data (see Section 3.1). In order to increase the signalto-noise ratio $(\mathrm{S} / \mathrm{N})$, a time binning of $100 \mathrm{~s}$ has been used. Special care has been taken to check that the target was well centered in the small fast-mode window in all exposures and to ensure that the background was properly measured.

\footnotetext{
5 The phases are calculated using the ephemeris by A2003 with phase 0 corresponding to periastron passage.
} 
Table 2

XMM-Newton Observations of AK Sco

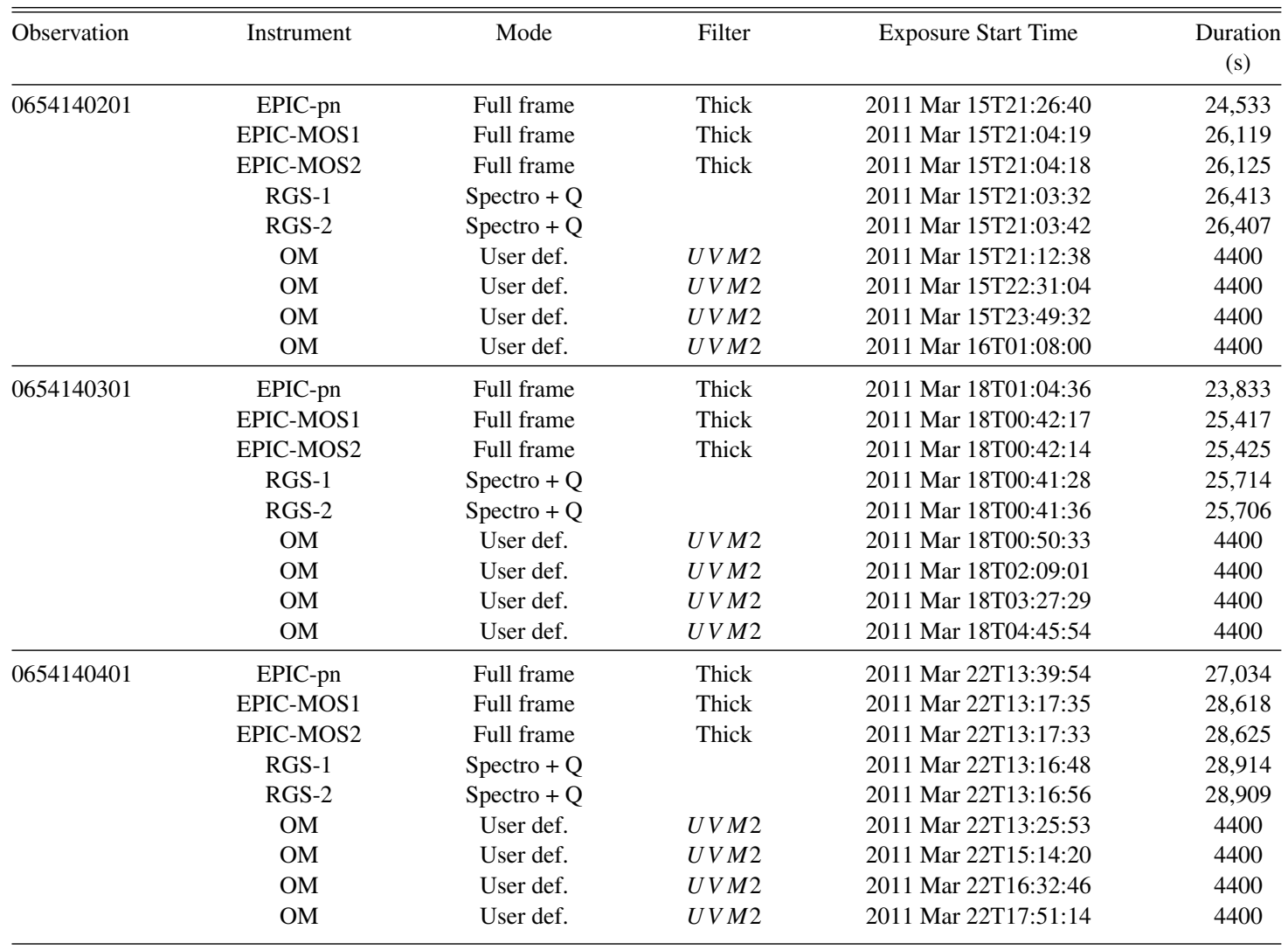

Table 3

Photometry of AK Sco with OM Using the $U V M 2$ Filter (2310 ̊)

\begin{tabular}{lcccc}
\hline \hline Observation & $\begin{array}{c}\text { HJD } \\
(-2400000.5)\end{array}$ & Phase & AB mag & $\begin{array}{c}\text { Flux } \\
\left(\mathrm{erg} \mathrm{s}^{-1} \mathrm{~cm}^{-2} \AA^{-1}\right)\end{array}$ \\
\hline 0654140201 & 55635.91003 & 0.9919 & 13.89 & $5.62 \mathrm{e}-14$ \\
& 55635.96450 & 0.9959 & 13.84 & $5.91 \mathrm{e}-14$ \\
& 55636.01900 & 0.9999 & 13.82 & $6.01 \mathrm{e}-14$ \\
& 55636.07350 & 0.0039 & 13.86 & $5.82 \mathrm{e}-14$ \\
\hline 0654140301 & 55638.06157 & 0.1500 & 13.74 & $6.48 \mathrm{e}-14$ \\
& 55638.11606 & 0.1540 & 13.74 & $6.45 \mathrm{e}-14$ \\
& 55638.17056 & 0.1580 & 13.79 & $6.19 \mathrm{e}-14$ \\
& 55638.22502 & 0.1620 & 13.79 & $6.17 \mathrm{e}-14$ \\
\hline 0654140401 & 55642.58653 & 0.4824 & 14.19 & $4.28 \mathrm{e}-14$ \\
& 55642.66185 & 0.4879 & 14.15 & $4.43 \mathrm{e}-14$ \\
& 55642.71633 & 0.4919 & 14.13 & $4.50 \mathrm{e}-14$ \\
& 55642.77082 & 0.4960 & 14.15 & $4.44 \mathrm{e}-14$ \\
\hline
\end{tabular}

\subsection{UV Archival Data of AK Sco}

The UV spectra of AK Sco obtained with the IUE have been retrieved from the IUE Newly Extracted Spectra (INES) archive for this study. The log of observations is in Table 4 (see Gómez de Castro \& Franqueira 1997 for further details). IUE observations were obtained with the long-wavelength prime (LWP) camera, long-wavelength redundant (LWR) camera, and the short-wavelength prime (SWP) camera in low-dispersion mode. The long-wavelength cameras obtained the spectrum in the $2000 \AA-3200 \AA$ spectral range and the SWP camera in the $1200 \AA-1950 \AA$ A range. IUE was equipped with a fine error sensor (FES) that was also used to provide estimates of the visual
Table 4

UV Observations of AK Sco

\begin{tabular}{|c|c|c|c|c|}
\hline \multicolumn{5}{|c|}{ IUE Observations } \\
\hline Observation & $\begin{array}{c}\text { HJD } \\
(-2400000.5)\end{array}$ & $\begin{array}{c}T_{\exp } \\
\text { (minutes) }\end{array}$ & Phase & FES $V$ mag \\
\hline \multicolumn{5}{|l|}{ Low dispersion } \\
\hline LWR14048 & 45210.77531 & 12 & 0.96 & 8.84 \\
\hline SWP17804 & 45210.85330 & 170 & 0.97 & 8.84 \\
\hline SWP33197 & 47252.82195 & 175 & 0.01 & 8.96 \\
\hline LWP12966 & 47252.94939 & 15 & 0.02 & 8.91 \\
\hline SWP33241 & 47260.78745 & 185 & 0.60 & 8.89 \\
\hline LWP13009 & 47260.93182 & 12 & 0.61 & 9.06 \\
\hline LWP13010 & 47260.97009 & 30 & 0.61 & 9.07 \\
\hline LWP13011 & 47261.01688 & 8 & 0.61 & 9.04 \\
\hline LWP28929 & 49583.48992 & 10 & 0.26 & $*$ \\
\hline \multicolumn{5}{|l|}{ High dispersion } \\
\hline LWP08847 & 46648.93805 & 281 & 0.64 & 9.1 \\
\hline LWP12964 & 47252.84456 & 85 & 0.01 & 8.9 \\
\hline LWP12967 & 47253.23681 & 560 & 0.04 & 8.9 \\
\hline LWP12968 & 47253.53749 & 165 & 0.06 & 8.9 \\
\hline LWP13006 & 47260.56819 & 430 & 0.58 & 9.0 \\
\hline \multicolumn{5}{|c|}{ HST Observations } \\
\hline Observation ID & $\begin{array}{c}\text { HJD } \\
(-2400000)\end{array}$ & Grating & $\begin{array}{l}T_{\exp } \\
\text { (s) }\end{array}$ & Phase \\
\hline ob6b21020 & 55430.26348 & E230M & 1015 & 0.84 \\
\hline ob6b21030 & 55430.27520 & E230M & 942 & 0.84 \\
\hline ob6b21040 & 55430.34160 & E140M & 2917 & 0.84 \\
\hline
\end{tabular}




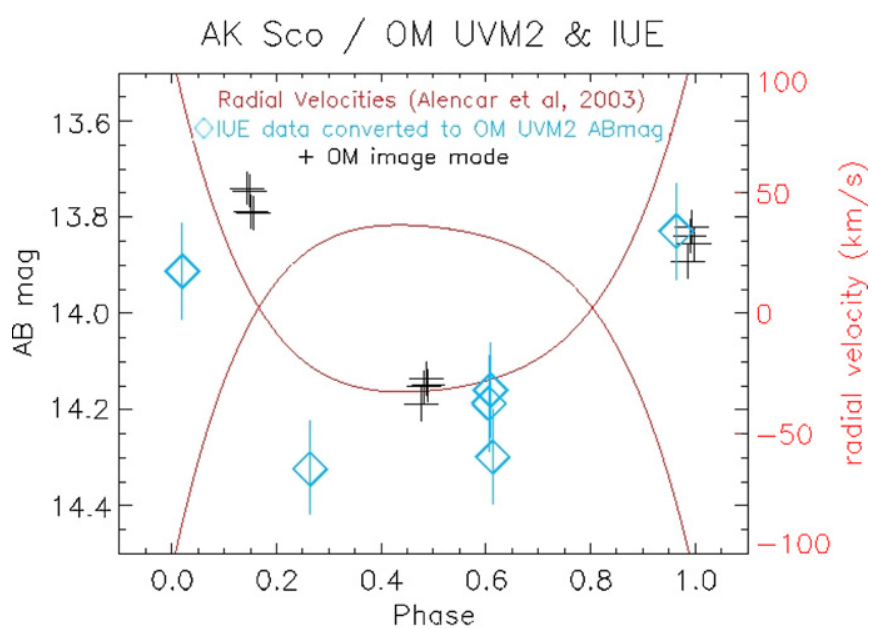

Figure 1. Observations of AK Sco with IUE and OM. AB magnitudes are overplotted on the radial-velocity curve as derived by A2003. Error bars are plotted for the $I U E$-based measurements. Error bars are negligible in this plot for OM observations.

(A color version of this figure is available in the online journal.)

magnitude with rms errors of 0.08 mag (Holm \& Rice 1981). The FES measurements have been converted into magnitudes using Stickland's (1980) and Pérez's (1991) calibration. They have been corrected for color effects, the sensitivity degradation of the cameras (Fireman \& Imhoff 1989) and the change of the FES reference point after 1990 January and July at the two ground control stations, NASA and European, respectively. FES magnitudes are also provided in Table 4 . AK Sco was observed with the IUE in low-dispersion ( $6 \AA$ resolution) and in highdispersion $(0.2 \AA$ resolution) modes. The binary was too weak to be observed in the high-dispersion mode below $\sim 2700 \AA$. As shown in Table 4, exposure times were of 6-9 hr. Only the $\mathrm{Mg}$ II lines are observed with a good enough $\mathrm{S} / \mathrm{N}$ (see Section 3.5).

\subsection{HST/STIS Archival Data of AK Sco}

Recently, high-S/N spectra of AK Sco have been obtained making use of the Space Telescope Imaging Spectrograph (STIS) on board the HST. The observations were obtained in echelle mode with gratings E230M $(R \sim 60,000)$ and E140M $(R \sim 91,000)$ on 2010 August 21 at phase 0.84 . The spectra cover the range $1150 \AA-3150 \AA$ in three observations obtained sequentially (see Table 4 for further details).

\section{DATA ANALYSIS}

\subsection{UV Photometry and Light Curves}

The results of the photometry with $\mathrm{OM}$ are displayed in Figure 1 where we plot the $\mathrm{AB}$ magnitudes in the $U V M 2$ filter (see Talavera 2011 for OM calibration). We plot here as well the integrated fluxes obtained from $I U E$ spectra in the OM- $U V M 2$ band, also as $\mathrm{AB}$ magnitude. All these data are overimposed in the radial-velocity curve from A2003. We see clearly how the fluxes are higher at periastron (phase 0.0 ), both in the OM and IUE data (note that IUE data were not obtained in the same binary system cycle than OM data). The OM band is sensitive to UV emission in the $2100 \AA-2800 \AA$ spectral range. AK Sco spectrum in this band is dominated by the Balmer continuum and the Fe II resonance multiplets (i.e., by plasma with an electron temperature of about $10^{4} \mathrm{~K}$; the so-called veiling spectrum); there are not strong spectral lines

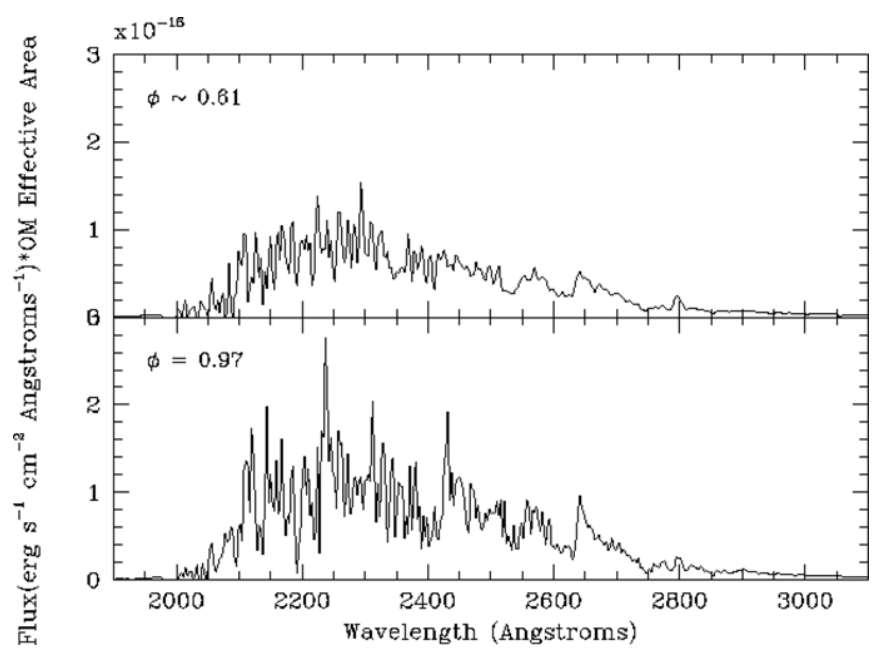

Figure 2. IUE UV spectrum of AK Sco convolved with OM-UVM2 response at two orbital phases. The flux is dominated by the resonance transitions of Fe II(uv1) (2585-2632 $\mathrm{A}), \mathrm{Fe}$ II(uv2) (2360-2410 $)$, and Fe II(uv3) (2327-2380 ̊) and the tail of the Balmer continuum.

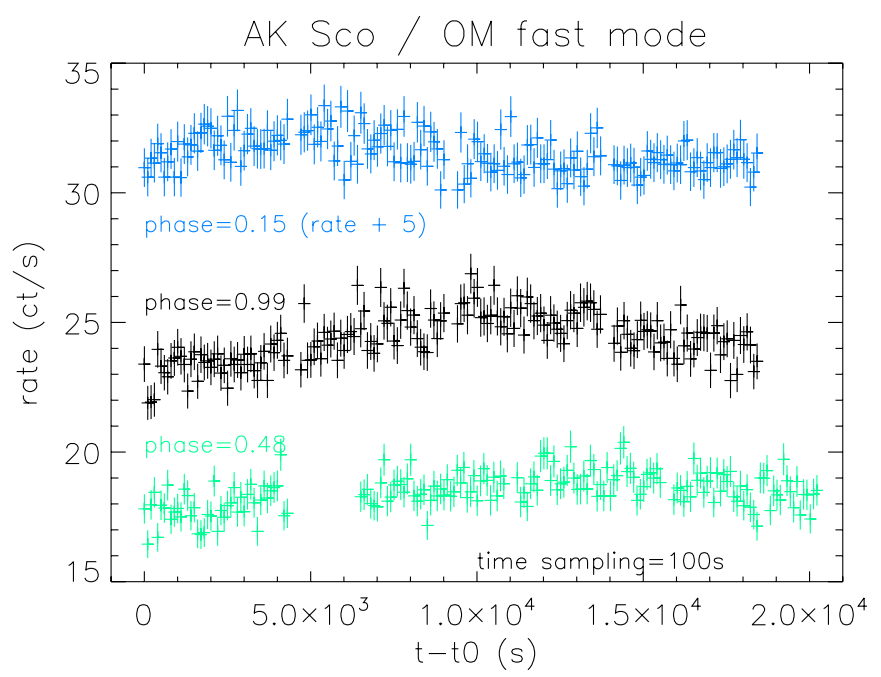

Figure 3. Light curves of AK Sco obtained with OM in fast mode. The light curve corresponding to phase 0.15 has been displaced by 5 counts $\mathrm{s}^{-1}$ upward for more clarity.

(A color version of this figure is available in the online journal.)

and the photospheric contribution is negligible. In Figure 2, the effective area of the $X M M-O M$ in the $U V M 2$ band has been multiplied by the UV spectrum of AK Sco (from IUE data; see Section 3.3) at two phases, showing the higher flux of the star at periastron, depicted also in Figure 1. Note that this veiling spectrum is a good indicator of the accretion rate in the TTSs (Ingleby et al. 2011).

The light curves from the three XMM-Newton observations with $\mathrm{OM}$ in fast mode are shown in Figure 3, where the abscissa is referred to the start of each observation. In addition to the variability with the phase shown in Figure 1 we see in the fastmode some short timescale variations along the duration of each observation. This short-term variability is analyzed in Gómez de Castro et al. (2013).

\subsection{Modeling the X-Ray Spectrum of AK Sco}

Spectra of the X-ray source were extracted from circles with radius 6 arcmin (this region contains $90 \%$ of the source photons, on-axis). Background spectra were extracted from 
Table 5

Parameters of the Fit for Each EPIC Observation

\begin{tabular}{|c|c|c|c|c|c|c|c|c|}
\hline ObsID & Phase & $\begin{array}{c}N_{\mathrm{H}} \\
\left(\times 10^{21} \mathrm{~cm}^{-2}\right)\end{array}$ & $Z / Z_{\odot}$ & $\begin{array}{c}k T \\
(\mathrm{keV})\end{array}$ & $\begin{array}{c}\mathrm{EM} \\
\left(\times 10^{52} \mathrm{~cm}^{-3}\right)\end{array}$ & $\chi_{\text {red }}^{2}($ dof $)$ & $\begin{array}{c}\text { Observed } f_{\mathrm{X}} \\
\left(\times 10^{-14} \mathrm{erg} \mathrm{cm}^{-2} \mathrm{~s}^{-1}\right)\end{array}$ & $\begin{array}{c}\log L_{X} \\
\left(\operatorname{erg~s}^{-1}\right)\end{array}$ \\
\hline \multicolumn{9}{|c|}{ All parameters varying freely } \\
\hline 0651870201 & 0.99 & $0.49_{-0.49}^{+0.64}$ & $0.27_{-0.09}^{+0.16}$ & $0.52_{-0.08}^{+0.06}$ & $1.1_{-0.6}^{+1.1}$ & $1.0(58)$ & $6.5_{-6.2}^{+1.1}$ & 28.9 \\
\hline 0651870301 & 0.15 & $0.08_{-0.08}^{+0.35}$ & $0.26_{-0.09}^{+0.13}$ & $0.58_{-0.04}^{+0.03}$ & $1.0_{-0.2}^{+1.6}$ & $1.0(62)$ & $7.3_{-2.7}^{+0.9}$ & 29.0 \\
\hline 0651870401 & 0.48 & $1.10_{-0.50}^{+0.68}$ & $0.13_{-0.05}^{+0.05}$ & $0.56_{-0.09}^{+0.05}$ & $1.6_{-0.6}^{+1.2}$ & $1.1(44)$ & $4.4_{-4.0}^{+0.7}$ & 28.7 \\
\hline \multicolumn{9}{|c|}{ Global abundance and temperature fixed } \\
\hline 0651870201 & 0.99 & $0.35_{-0.29}^{+0.29}$ & $=0.26$ & $=0.55$ & $1.1_{-0.1}^{+0.2}$ & $1.0(60)$ & $6.6_{-0.9}^{+1.0}$ & 28.9 \\
\hline 0651870301 & 0.15 & $0.23_{-0.26}^{+0.23}$ & $=0.26$ & $=0.55$ & $1.1_{-0.1}^{+0.1}$ & $1.0(64)$ & $7.1_{-1.0}^{+0.9}$ & 29.0 \\
\hline 0651870401 & 0.48 & $1.11_{-0.46}^{+0.49}$ & $=0.26$ & $=0.55$ & $1.0_{-0.2}^{+0.2}$ & $1.2(46)$ & $4.2_{-0.9}^{+0.7}$ & 28.7 \\
\hline
\end{tabular}

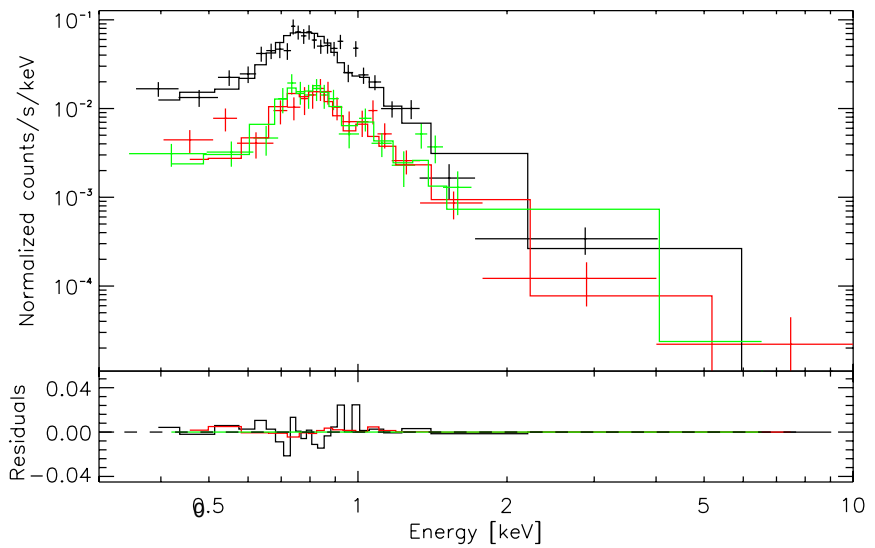

Figure 4. Data and folded model for EPIC observation ID.0654140201 of AK Sco. Data are marked with crosses and models with lines. Colors are used to code EPIC-PN data (black), EPIC-MOS1 (red), and EPIC-MOS2 (green).

(A color version of this figure is available in the online journal.)

a nearby region of the same chip, free of any other X-ray source. Response matrices and ancillary files were created for each observation. The analysis of the spectra was done with XSPEC ${ }^{6}$ (Arnaud 1996, 2004). Independently for each observation, the background-corrected source spectrum was fit against a hot plasma model. We chose the model by Smith et al. (2001a), which uses the atomic data contained in the Astrophysical Plasma Emission Database (APED; Smith et al. 2001b) and it is available in XSPEC. A multiplicative interstellar absorption model - in particular the one described in Morrison \& McCammon (1983) — was added to account for the possible effects of interstellar or circumstellar material of the source $\mathrm{X}$-ray spectrum. Therefore, the parameters of the fit were the hydrogen column density $\left(N_{\mathrm{H}}\right)$, the plasma temperature $(k T)$, the abundance (referred to the solar photosphere, $Z / Z_{\odot}$ ), and the normalization constant, which is directly related to the emission measure (EM). Energy bins were grouped to contain at least 25 counts each.

A first attempt of fitting was done allowing all the parameters to vary. The results of the fit for each observation are shown in Table 5; the fit of observation ID.0654140201 is shown in Figure 4. Errors were determined with XSPEC at a confidence level of $90 \%$, following the recipes of the XSPEC manual. ${ }^{7}$ No clear differences in the temperature or global abundance are observed in the three orbital phases. However, $N_{\mathrm{H}}$ vary notably

\footnotetext{
$6 \mathrm{http} / / /$ heasarc.nasa.gov/xanadu/xspec/

7 http://heasarc.nasa.gov/xanadu/xspec/xspec11/manual/manual.html
}

at phase 0.48 . The total X-ray flux also varies with the orbital phase being maximum at periastron and minimum at apastron.

It is well known that in the limit of low counts, the column density and the global abundance are degenerated for the fit (Arnaud 2004; Albacete Colombo et al. 2007). Therefore, changing the value of any of them will affect the result for the other. In order to check this effect in our data, we studied the space of parameters in the model, using the command steppar in XSPEC. For $N_{\mathrm{H}}$, we obtained that its value is slightly correlated with $Z / Z_{\odot}$, while for temperature there is no such a correlation. Then, we decided to fix the global abundance, since we see little or no change in its value during the three phases covered by our data. We used $Z / Z_{\odot}=0.26$, the value determined from the first two observations. The temperature was also fixed to the mean value determined from the three orbital phases $(k T=0.55 \mathrm{keV}$; $\left.T \sim 6.4 \times 10^{6} \mathrm{~K}\right)$. Then, a new fit to the data was performed for each observation. The results of the new fits are shown also in Table 5. We see that the increase in $N_{\mathrm{H}}$ persists, while the little change detected in the EM for the third observation disappears when $k T$ and $Z / Z_{\odot}$ are fixed.

To investigate variations of the X-ray emission level of the source with time, we obtained time-binned light curves by extracting the counts from the same regions than those used to extract the spectra (for both source and background) in the energy range [0.3-10] keV. Unfortunately, the count rates are very low and therefore no variability can be detected in short time periods (of the order of few kiloseconds). However, we found the mean count rate in this energy band to be variable and it varies as the UV count rate measured with the OM (see Section 3.1). We note that high-frequency (stochastic) events, i.e., flares, may be taking place during the observations. However, the low count rate shown by the star prevents any detection of small flares. Large flares are much less frequent and the probability of detecting one during short observations is very low. Nevertheless, the low temperature detected during each observation does not suggest the presence of any kind of flaring event during our observations.

\subsection{Analysis of the IUE Data in Low Resolution}

The UV spectrum of AK Sco is dominated by the photospheric contribution at long wavelengths, as expected in an F5 star (see Figure 5). At shorter wavelengths the spectrum is dominated by the tail of the Balmer continuum and some prominent emission lines. In the near UV (2200-3200 ̊), the dominant transitions are the Fe II and the $\mathrm{Mg}$ II resonance multiplets. In the far UV (1200-1950 $)$, the most prominent features are the resonance lines of $\mathrm{HI}(\mathrm{Ly} \alpha), \mathrm{N}$ v[uv1], O I[uv1], C II[uv1], 
Table 6

UV Line Fluxes from IUE Spectra

\begin{tabular}{lccccccc}
\hline \hline Phase & O I & C II & Si IV & C IV & Si III & C III & Mg II \\
\hline \multicolumn{7}{c}{$\left(\times 10^{-13} \mathrm{erg} \mathrm{s}^{-1} \mathrm{~cm}^{-2}\right)$} \\
\hline 0.97 & $(2.8 \pm 0.5)$ & $(1.8 \pm 0.3)$ & $(2.3 \pm 0.5)$ & $(5.5 \pm 0.5)$ & $(3.5 \pm 0.3)$ & $(2.1 \pm 0.2)$ & $(19 \pm 3)$ \\
$\sim 0.02$ & $(2.2 \pm 0.4)$ & $(2.2 \pm 0.4)$ & $(2.3 \pm 0.6)$ & $(5.9 \pm 0.7)$ & $(2.7 \pm 0.3)$ & $(1.8 \pm 0.2)$ & $(21 \pm 3)$ \\
$\sim 0.60$ & $(4.1 \pm 0.9)$ & $(2.8 \pm 0.7)$ & $(2.6 \pm 1.2)$ & $(5.6 \pm 0.8)$ & $(3.1 \pm 0.3)$ & $(2.4 \pm 0.3)$ & $(26 \pm 3)$ \\
\hline
\end{tabular}

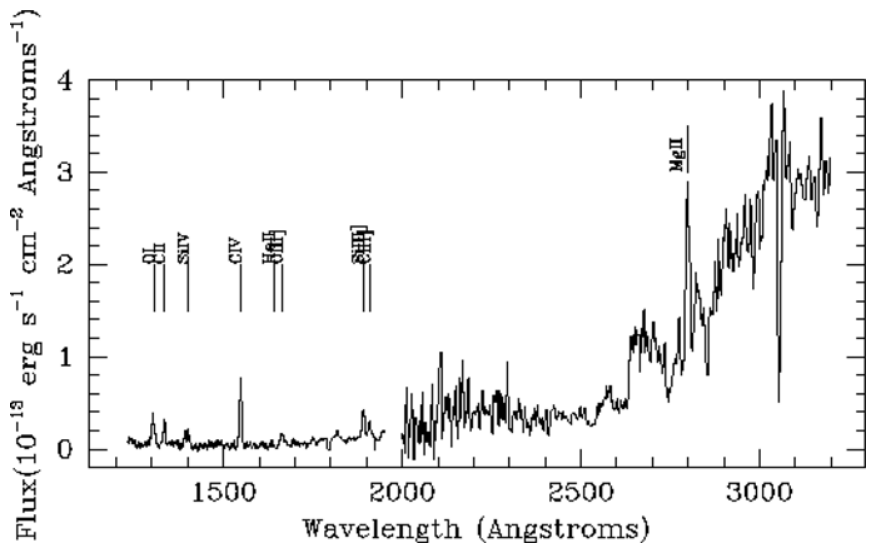

Figure 5. Low-dispersion $I U E$ spectrum of AK Sco.

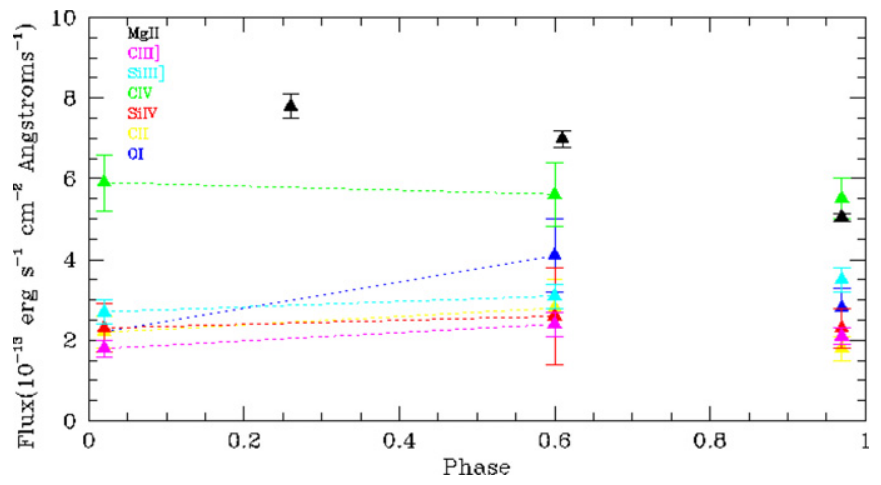

Figure 6. Variation of the fluxes of the main spectral lines in the UV spectrum of AK Sco, from low-dispersion IUE data.

(A color version of this figure is available in the online journal.)

Si Iv[uv1], and C Iv[uv1] and the nebular, semiforbidden transitions of O III] (1666 ̊), Si III] (1892 Å), and C III] (1908 ̊̊).

IUE low-resolution observations covered a broad range of phases. Spectra LWR14048 and SWP17804 were obtained at phase 0.97, spectra LWP12966 and SWP33197 at phase $\sim 0.02$, and spectra LWP 13009-13011 and SWP33241 at phase $\sim 0.61$. The observations corresponding to the last two phases were obtained during the same cycle (see Table 4). The line fluxes measured in the IUE spectra are given in Table 6. They have been extinction corrected using Valencic et al. (2004) extinction law, with $A_{V}$ and $R$ as in Table 1 . The variation of the line flux is shown in Figure 6. Note that for rather similar phases $(0.97$ and 0.02) some lines display significant variations (see also Table 6). However, for the observations obtained at different phases (periastron and apastron) significant flux variations are detected in the $\mathrm{O}_{\mathrm{I}}$ line, and to a smaller degree in the the $\mathrm{C}$ III] semiforbidden transition. These two lines are bona fide tracers of nebular (extended) emission in the TTS environment (see, e.g., Gómez de Castro \& Verdugo 2001 for the C III] emission). Note also that the OI line may be excited either by the Bowen mechanism from $\operatorname{H~Ly} \beta$ (Bowen 1947) or in
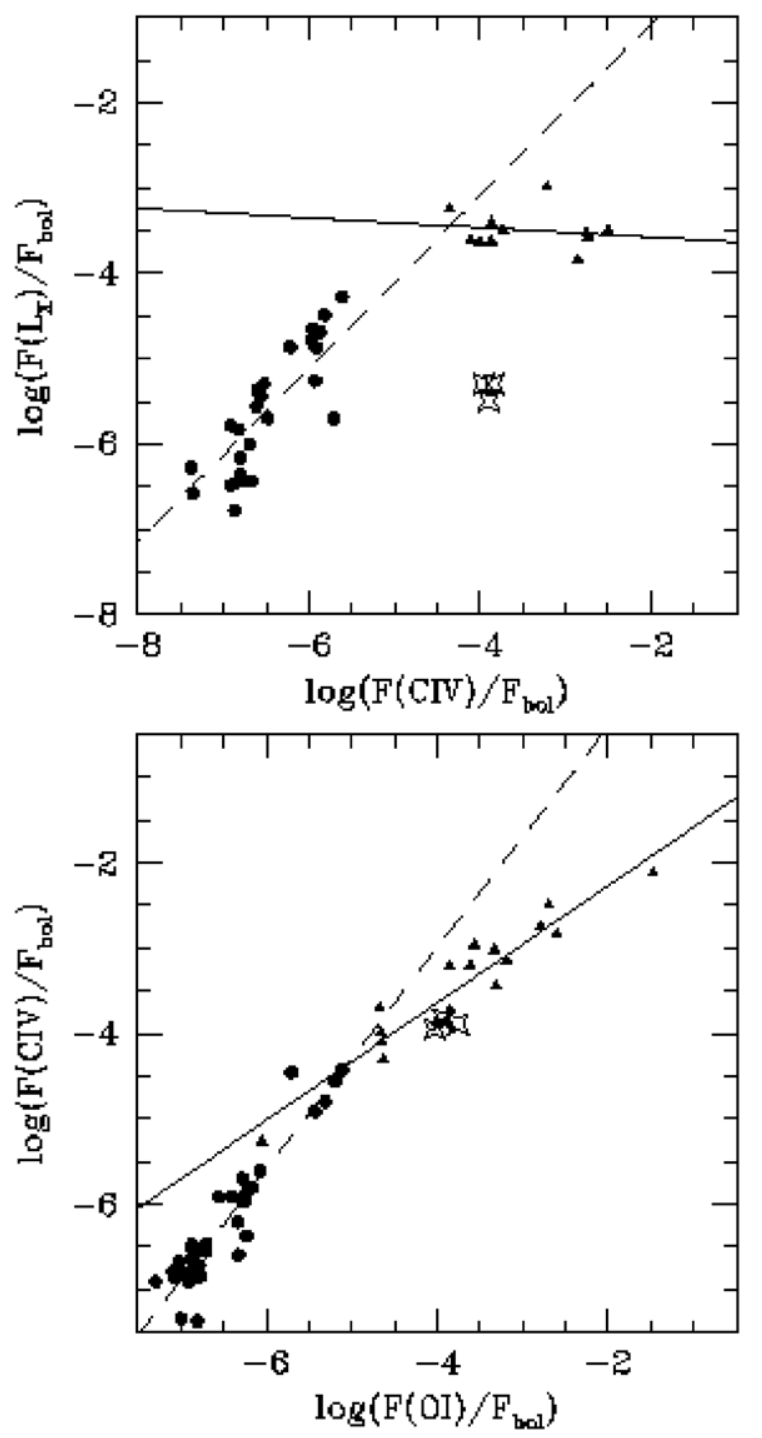

Figure 7. Location of AK Sco in the flux-flux diagrams of cool main-sequence stars and TTSs as in GdCMA2012. Top: X-ray vs. Civ; bottom: Civ vs. O I. The filled circles represent the location of late-type main-sequence stars in the diagram from Ayres et al. (1995) and Linsky et al. (1982). The filled triangles represent the location of TTSs from GdCMA2012. The regression lines for late-type stars and TTSs are represented by dashed and solid lines, respectively. The location of AK Sco is marked with stars.

the recombination of $\mathrm{O}$ II. In both cases, the line excitation is expected to trace the end of the recombination cascade following photoionization by the stellar ionizing radiation field, as suggested by GdCMA2012. In this context, the enhancement of the $\mathrm{O}_{\mathrm{I}}$ flux at apastron would suggest a more efficient illumination of the circumbinary disk when the distance between both stars is the largest.

In Figure 7, the line fluxes have been normalized to the bolometric flux and represented in the standard flux-flux diagrams 
for the TTS atmospheric/magnetospheric diagnostics revised recently by GdCMA2012. AK Sco is located on the TTS regression line in the CIV versus O I diagram. It displays the characteristic excess emission of neutrals compared to high ionization species. This excess is partially caused by the reprocessing of stellar UV photons in the inner disk (GdCMA2012). However, AK Sco stands out of the TTS regression line in the $\mathrm{X}$-ray versus $\mathrm{C}$ IV diagram. The source of this X-ray luminosity defect is unclear. The X-ray luminosities of the TTSs plotted in the diagram have been derived from the XEST survey (Guedel et al. 2007), i.e., from XMM-Newton observations, as the AK Sco data. The TTSs in the plot belong to spectral types from G0 to M5; their X-ray/UV excess was found not to depend on the spectral type (GdCMA2012). For comparison, the X-ray/UV data of the main-sequence stars have been plotted. Data have been extracted from Ayres et al. (1995) for G to K types and from Linsky et al. (1982) for M stars. The X-ray/UV excess of main-sequence stars depends strongly on the spectral type with the largest excess being observed in the latest types. Ayres et al. (1995) extended this study to F-type stars and showed that the correlation is broken at late F types; this is assumed to be caused by a decay in the dynamo activity since F-type stars represent an intermediate regime between stars with convective envelopes and stars with radiative envelopes. Typically, the $\mathrm{X}$-ray luminosity of F8-F0 main-sequence stars is about an order of magnitude weaker than predicted by the main-sequence regression line. AK Sco data seem to extend this behavior to PMS stars in a more dramatic manner: the X-ray flux drops by two orders of magnitude in spite of the strong tides. In this context we should remember that the X-ray fluxes of other PMS close binaries are also found to be unexpectedly weak; DQ Tau, UZ Tau, and KH $15 \mathrm{D}$ also seem to be subluminous in X-rays when compared with main-sequence stars of the same spectral types (Herbst \& Moran 2005 and references therein). All the components in these systems are late-type stars (K-M) and should not be affected by the transition between convective and radiative envelopes. There are, however, PMS-SBs such as V826 Tau with normal X-ray luminosities (Carkner et al. 1996).

\subsection{HST/STIS Spectra}

The high sensitivity of HST has allowed us to obtain for the first time good $\mathrm{S} / \mathrm{N}$ profiles of the main spectral lines in the UV spectrum of AK Sco. The profiles of the most prominent lines (Si III (1206 ̊), Ly $\alpha$, N v[uv1], O I[uv1], C II[uv1], Si Iv[uv1], C Iv[uv1], O III] (1666 ̊), Si III (1892 A), C III (1908 A), and $\mathrm{Mg}$ II[uv1]) are displayed in Figure 8; all but the $\mathrm{C}$ III] line have $\operatorname{good} \mathrm{S} / \mathrm{N}$ in the $H S T$ observations.

The profiles are very broad and asymmetric, suggesting that line emission is produced in extended, unresolved structures. The $\mathrm{Mg}$ II doublet is saturated since the $2796 / 2803$ line ratio is $\sim 1.4$ instead of 2; however, hot plasma tracers such as the Si IV and CIv[uv1] doublets have line ratios close to their values in optically thin plasma. The blue edge velocity in the nonsaturated profiles is $\sim 350 \mathrm{~km} \mathrm{~s}^{-1}$. This value corresponds to the Keplerian velocity at $\sim 1.8 R_{*}$ which according to Gómez de Castro (2009b) corresponds roughly to the extent of the emission region.

It is also noticeable the large broadening of the $\mathrm{N} \mathrm{v}$ line, with a FWHM of $278.7 \mathrm{~km} \mathrm{~s}^{-1}$. $\mathrm{N} \mathrm{v}$ is typically excited in the transition region of the cool star atmospheres at temperatures above some $40,000 \mathrm{~K}$. As the thermal speed of the plasma
Table 7

Characteristics of the UV Lines Observed with HST/STIS

\begin{tabular}{|c|c|c|c|}
\hline Line & $\begin{array}{l}\text { Wavelength } \\
(\AA)\end{array}$ & $\begin{array}{c}\text { Flux }^{\mathrm{a}} \\
\left(\times 10^{-14} \mathrm{erg} \mathrm{s} \mathrm{cm}^{-2}\right)\end{array}$ & $\begin{array}{l}\text { FWHM } \\
\left(\mathrm{km} \mathrm{s}^{-1}\right)\end{array}$ \\
\hline Si III & 1206.5 & $11.4 \pm 1.9$ & 400.2 \\
\hline $\mathrm{Nv}$ & 1238.8 & $2.1 \pm 0.5$ & 278.7 \\
\hline $\mathrm{NV}$ & 1242.8 & $0.6 \pm 0.3$ & $\ldots$ \\
\hline $\mathrm{OI}_{\mathrm{I}}$ & $1302,1305,1306$ & $13.7 \pm 1.8$ & $\ldots$ \\
\hline $\mathrm{C}_{\text {II }}$ & 1335,1336 & $13.8 \pm 0.9$ & $\ldots$ \\
\hline Si IV & 1393.8 & $8.2 \pm 1.1$ & 404.6 \\
\hline Si IV & 1402.8 & $9.5 \pm 1.2$ & 418.2 \\
\hline C IV & 1548,1550 & $35.4 \pm 2.7$ & $\ldots$ \\
\hline O III & 1666 & 3.3 & $\ldots$ \\
\hline Si III & 1892.03 & 16.0 & 219 \\
\hline Mg II & 2796,2803 & $262 . \pm 18$ & $\ldots$ \\
\hline \multicolumn{4}{|c|}{$\mathrm{H}_{2}$ lines } \\
\hline $\mathrm{P}(2) 0-4$ & 1338.554 & 0.35 & 56.1 \\
\hline $\mathrm{P}(3) 0-4$ & 1342.301 & 0.18 & 29.1 \\
\hline $\mathrm{P}(2) 0-5$ & 1399.013 & 0.29 & 34.0 \\
\hline $\mathrm{R}(3) 1-6(?)$ & 1430.918 & 0.26 & $\ldots$ \\
\hline $\mathrm{P}(5) 1-7$ & 1504.756 & 0.69 & 43.9 \\
\hline $\mathrm{P}(8) 1-7$ & 1524.597 & 0.27 & 31.5 \\
\hline $\mathrm{R}(11) 2-8$ & 1556.803 & 0.19 & 28.2 \\
\hline $\mathrm{P}(5) 1-8$ & 1562.365 & 0.33 & 36.5 \\
\hline
\end{tabular}

Note. ${ }^{\text {a }}$ Fluxes are not extinction corrected.

at this temperature is $25 \mathrm{~km} \mathrm{~s}^{-1,8}$ the large broadening has to be caused by unresolved high velocity motions, confirming the existence of disturbed magnetospheres in AK Sco stellar components (Gómez de Castro 2009b).

The red wing of the profiles is weaker than the blue wing and seems to be partially absorbed in the resonance multiplets (Si III, O I, C II, Si IV, C IV, and MgII). However, the profiles corresponding to semiforbidden transitions of $\mathrm{O}_{\mathrm{III}}$ ] and $\mathrm{Si}$ III] are also asymmetric, suggesting an inherent asymmetry of the profiles. The asymmetry indicates that the warm matter close to the stars is unevenly distributed in a high velocity field like the expected, for instance, in spiral structures (see Section 4).

The Ly $\alpha$ profile is completely different from the rest of the profiles since the blue wing is fully absorbed by the Lyman $\mathrm{H}_{2}$ band (Herczeg et al. 2002) and also, presumably, by a cool neutral outflow that it is not detected in the $\mathrm{Mg}$ II line. The strongest $\mathrm{H}_{2}$ transitions pumped by Ly $\alpha$ are detected in the spectrum (see Figure 8 and Table 7). The $\mathrm{H}_{2}$ line broadening is $\sim 30 \mathrm{~km} \mathrm{~s}^{-1}$ that corresponds to a thermal velocity of about $10^{4} \mathrm{~K}$, characteristic of the photoevaporative flows from protostellar disks.

\subsection{Variations in the Mg II Profiles from IUE and HST}

The Mg II profile varies significantly in the four observations available (see Figure 9); however, it does not seem to be a systematics when profiles corresponding to different cycles are compared (compare the profiles obtained at phases 0.56 and 0.64). This fact was already reported by A2003 for the $\mathrm{H} \alpha$ line that it is excited in similar physical conditions than the $\mathrm{Mg}$ II doublet; the $\mathrm{H} \alpha$ profile at periastron is observed to be an inverse $\mathrm{P}$ Cygni profile in one cycle and a double-peaked profile in

8 The ion thermal velocity of fully ionized plasma at $40,000 \mathrm{~K}$ is $v_{\text {th }}=\left(k_{B} T_{i} / m_{i}\right)^{1 / 2}$, with $k_{B}$ the Boltzmann constant and $T_{i}$ and $m_{i}$ the ion temperature and mass, respectively. For $T_{i}=40,000 \mathrm{~K}$ and $m_{i}=\mu m_{\mathrm{H}}$, $v_{\text {th }}=25 \mathrm{~km} \mathrm{~s}^{-1}$, where the mean molecular weight $\mu$ is 0.536 , as corresponds to hot (fully ionized) plasma with solar abundances. 

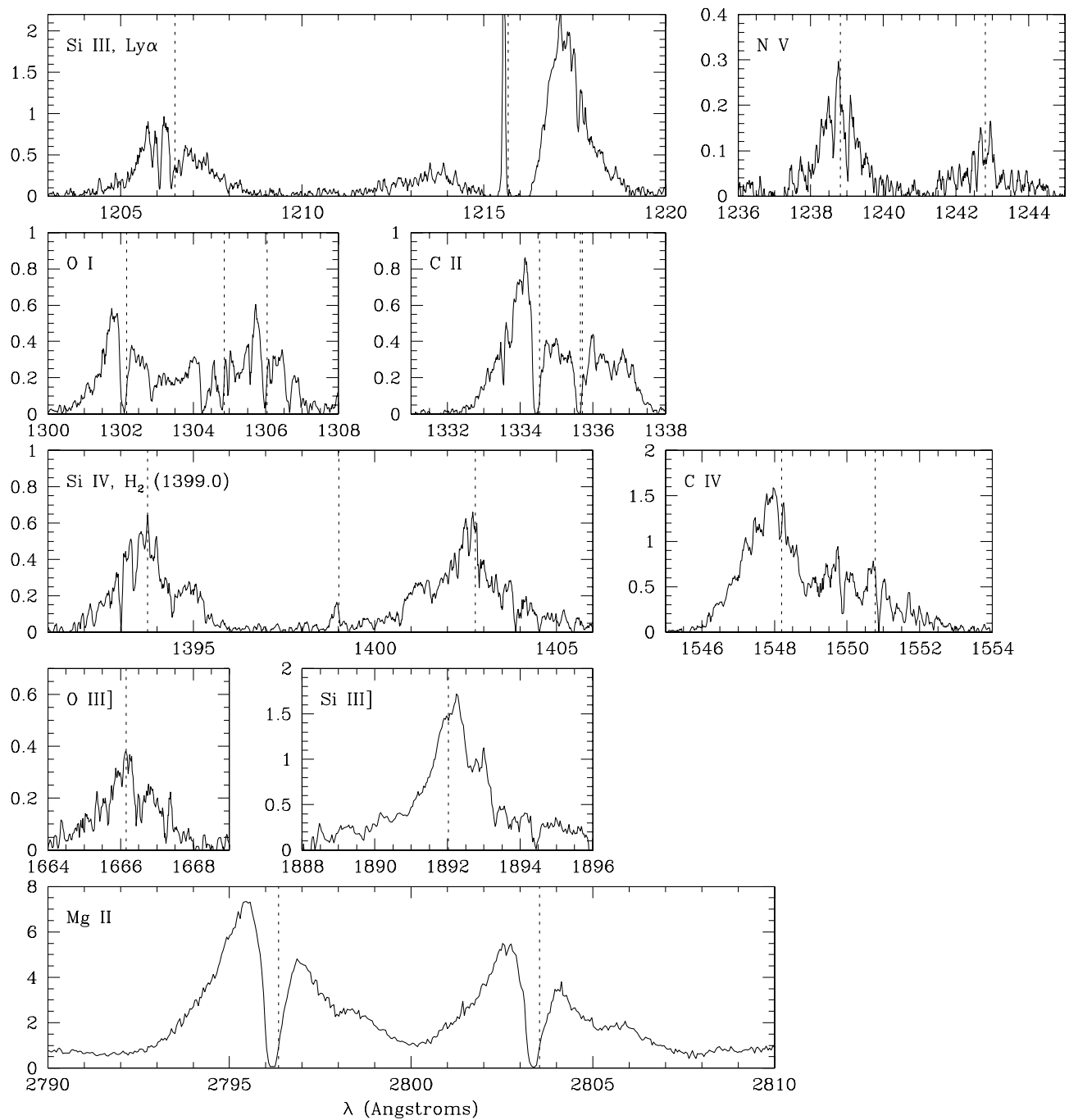

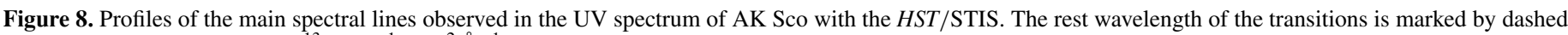
lines. Fluxes are in units of $10^{-13} \mathrm{erg} \mathrm{s}^{-1} \mathrm{~cm}^{-2} \AA^{-1}$.

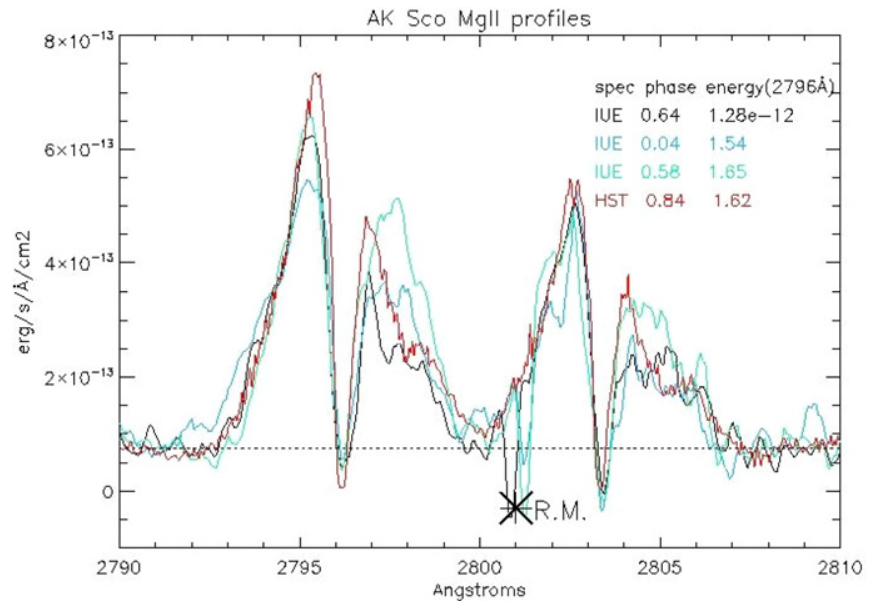

Figure 9. Variability of the $\mathrm{Mg}$ II profiles of $\mathrm{AK}$ Sco from the high-dispersion spectra in the IUE and HST archives. RM denotes an IUE fiducial reseau mark contaminating the spectrum at $\sim 2801 \AA$.

(A color version of this figure is available in the online journal.)

another cycle. It is, however, expected that the gas flow dragged from the inner border of the circumbinary disk contributes significantly to the formation of the lines and henceforth that the line profiles of warm (few thousand kelvins) plasma tracers, such as the $\mathrm{Mg}$ II or the $\mathrm{H} \alpha$ line, depend on the orbital phase.

In this context, Hamilton et al. (2012) have reported a correlation between the $\mathrm{H} \alpha$ profile variations and the orbital phase in the KD 15D. In this system, the $\mathrm{H} \alpha$ profile changes from an inverse P Cygni during ingress (as the stars approach the periastron) to a double-peaked profile during egress (stars move away from periastron passage). Unfortunately, the $\mathrm{Mg}$ II profiles of AK Sco are few and obtained at singular points in the orbit, either at the periastron (phase 0.04 ) or very close to apastron (phases 0.58 and 0.64) and there is not any clear trend between the profile asymmetry (the relative strength of the red to blue emission peaks) and the orbital phase (see Figure 9).

\section{NUMERICAL SIMULATIONS OF THE BINARY-DISK INTERACTION}

Numerical simulations of the evolution of disks around PMS binaries show that the circumbinary disk is formed with an inner gap of radius about 2-3 times the semimajor axis of the orbit (Artymowicz \& Lubow 1994; Bate \& Bonnel 1997; Guenther \& Kley 2002; Hanawa et al. 2010; Kaigorodov et al. 2010; Fateeva et al. 2011; de Val-Borro et al. 2011; Shi et al. 2012). In addition, stellar accretion disks may be formed around each component of the system depending on the flow rate through the inner disk gap 


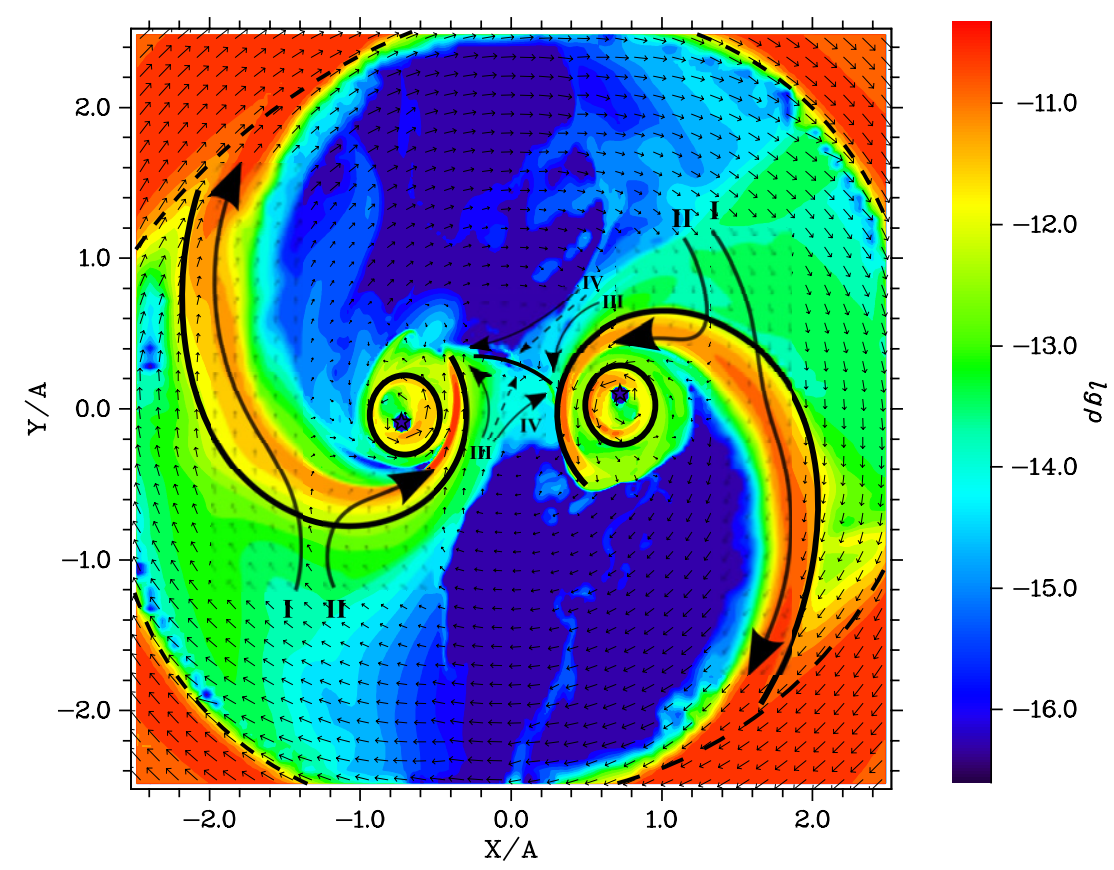

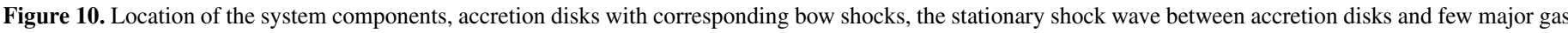

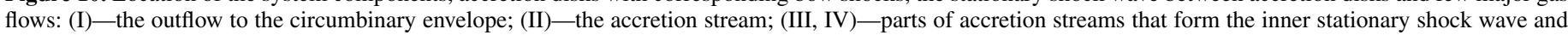
contribute to the accretion rates. The dashed line indicates the boundary of the gap.

to the stars and the properties of the binary system. To assist the interpretation of the X-ray, UV flux modulation, and the broad non-thermal motions unveiled by the UV spectra, we have run a set of numerical simulations of the interaction between the binary and the circumbinary disk.

The details of the numerical scheme used in this paper are described in Bisikalo et al. (2000) and Sytov et al. (2011). The parameters of the system are listed in Table 1. The evolution of the system is parameterized in terms of the orbital phase as defined by the ephemeris, the rotation was assumed counterclockwise, and the phase $\phi=0$ corresponds to the periastron. In this problem setup, the positions of the components are defined by expressions

$$
\begin{aligned}
& \boldsymbol{r}_{1}=\left[\left(1-q^{\prime}\right)(\cos (\theta)-e) A, \quad\left(1-q^{\prime}\right) \sqrt{1-e^{2}} \sin (\theta) A, \quad 0\right] \\
& \boldsymbol{r}_{2}=\left[-q^{\prime}(\cos (\theta)-e) A, \quad-q^{\prime} \sqrt{1-e^{2}} \sin (\theta) A, \quad 0\right],
\end{aligned}
$$

where $q^{\prime}=(q / q+1)$, and $q=\left(M_{1} / M_{2}\right)$ is the mass ratio of the components, $A$ is the semimajor axis, $\theta$ is the phase angle of the binary system, and the phase $\phi$ is defined as $\phi=\theta / 2 \pi$. For a given time $(t)$ the angle $\theta$ is calculated from the implicit relation $\theta=\left(2 \pi / P_{\text {orb }}\right) t+e \sin (\theta)$.

The gas flow is described by the system of Euler equations:

$$
\begin{aligned}
\frac{\partial \rho}{\partial t}+\nabla \rho \boldsymbol{v} & =0 \\
\frac{\partial \rho \boldsymbol{v}}{\partial t}+\nabla(\rho \boldsymbol{v} \otimes \boldsymbol{v})+\nabla P & =-\rho \nabla \Phi \\
\frac{\partial}{\partial t}\left(\rho\left(\varepsilon+\frac{v^{2}}{2}\right)\right)+\nabla \rho \boldsymbol{v}\left(\varepsilon+P / \rho+\frac{v^{2}}{2}\right) & =-\rho \boldsymbol{v} \nabla \Phi
\end{aligned}
$$

where $\rho$ is the gas density, $\boldsymbol{v}$ is the velocity vector, $\varepsilon$ is the internal energy, and $P$ is the gas pressure. An adiabatic equation of state is assumed with $P=(\gamma-1) \rho \varepsilon$ with $\gamma=1.01$ to keep the solution close to the isothermal one. The variable gravitational potential, $\Phi$, is given by the following expression:

$$
\Phi=-\frac{G M_{1}}{\left|\boldsymbol{r}-\boldsymbol{r}_{1}\right|}-\frac{G M_{2}}{\left|\boldsymbol{r}-\boldsymbol{r}_{2}\right|}
$$

The system of gas-dynamic equations is solved numerically using the Roe-Osher-Einfeldt scheme (see Boyarchuk et al. 2002 and references therein).

\subsection{The Disk Model}

The circumbinary disk is considered as a Keplerian disk with constant equatorial density and hydrostatic vertical density profile:

$$
\begin{gathered}
\rho(r, z)=\rho_{d} \exp \left(-\left(\frac{|z|}{h(r)}\right)^{2}\right), \\
h(r)=\frac{c_{s}}{\Omega(r)}, \quad \Omega(r)=\sqrt{G\left(M_{1}+M_{2}\right) / r^{3}},
\end{gathered}
$$

where $c_{s}$ is the sound speed, $\Omega(r)$ is the angular velocity of the matter in the circumbinary dirk, $\rho_{d}$ is the density in the orbital plane, and $r$ is the distance to the center of mass of the binary system. The temperature was assumed to be constant over the disk.

For all simulations we have run, the equatorial density and temperature of the disk were set to $\rho_{d}=10^{-11} \mathrm{~g} \mathrm{~cm}^{-3}$ and $T_{d}=1000 \mathrm{~K}$, respectively.

The most important parameter of the numerical model is the value of the effective viscosity, $\alpha$. The effective viscosity (including contribution of the scheme, grid, waves, etc.) determines the rate of the angular momentum transfer in the disk, radial velocity $v_{r}$, and, hence, the accretion rate, $\dot{M}_{a}$. The value of effective viscosity in the simulations was equal to $\sim 0.01$, which is in a good agreement with observations of accretion disks in cataclysmic variables. We use the boundary value of density $\rho_{b}$ to fit the numerical results to the observations, assuming that 

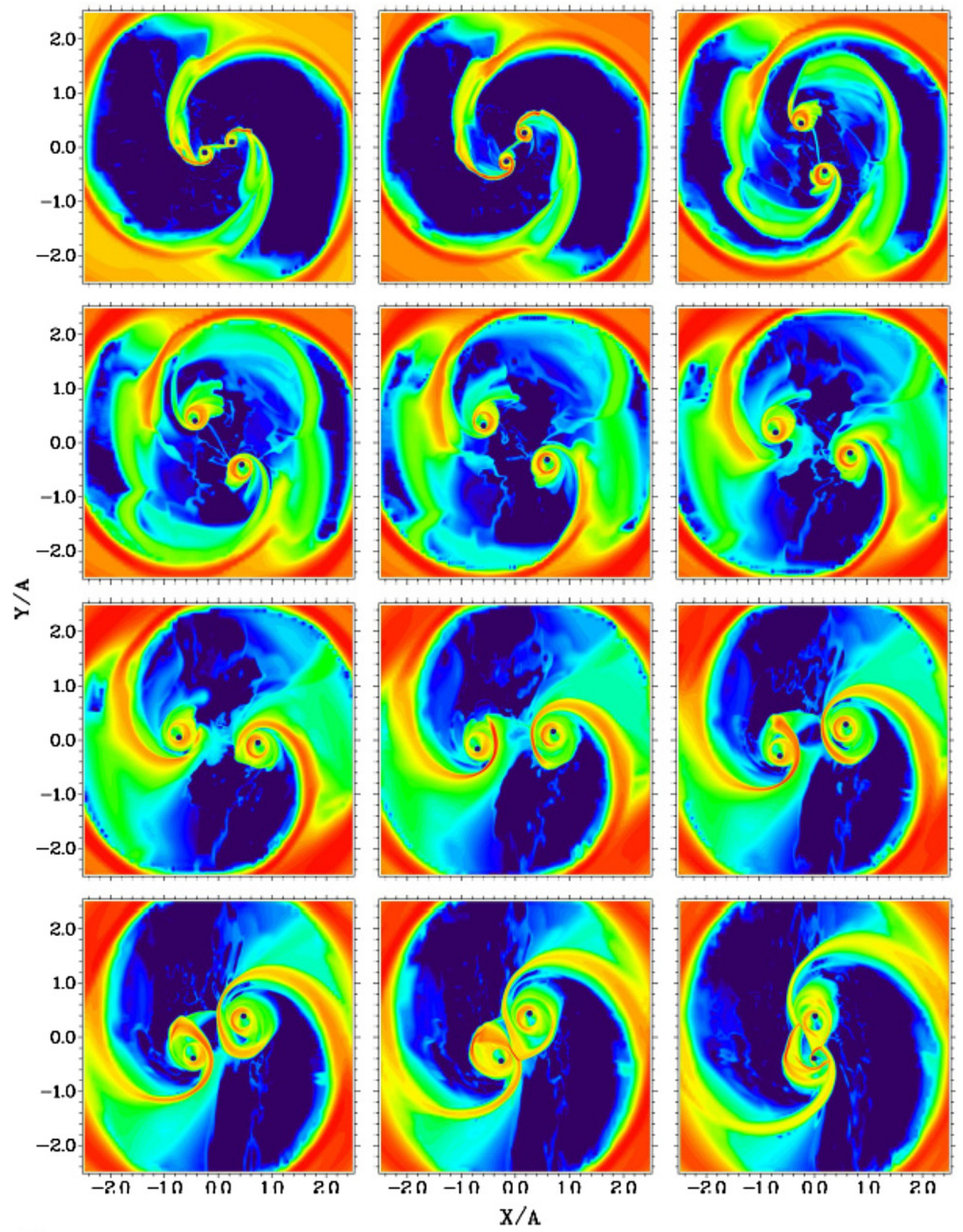

$l g \rho$
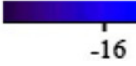

$-15$

$-14$

$-13$

$-12$

$-11$

$-10$

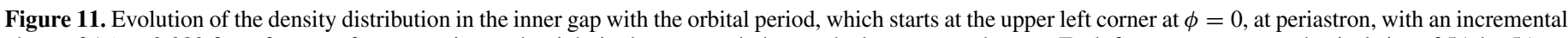
phase of $\Delta \phi=0.083$ from frame to frame running to the right in the rows and, then to the bottom, row by row. Each frame represents a physical size of $5 \mathrm{~A}$ by $5 \mathrm{~A}$.

$\alpha\left(\right.$ and $\left.v_{r}\right)$ is constant over the disk. Indeed, in the steady-state regime the accretion rate $\dot{M}_{a}$ equals the matter inflow through the outer boundary $\dot{M}$; thus, $\rho_{b}$ can be evaluated using the observed accretion rate. Unfortunately, the accretion rate is not well determined from the observations of AK Sco. The infrared spectral energy distribution can be properly fit only assuming reprocessing of the stellar radiation in the disk (A2003) setting up an upper limit to the accretion flow of $\sim 10^{-8} M_{\odot} \mathrm{yr}^{-1}$. However, this value seems to be too high for the observed UV excess of AK Sco; thus, a fiducial accretion rate of $10^{-9} M_{\odot} \mathrm{yr}^{-1}$ has been used.

\subsection{The Computational SetUp}

The computational domain is a box of size: $12 A \times 12 A$ in the orbital plane (plane $X Y$ ) by $\pm 0.75 A$ above/below it, in the
$Z$-axis or vertical direction. The center of the Cartesian coordinate system is at the center of mass of the binary. The motion of the binary system in the $X Y$ plane is set to be counterclockwise. The domain is covered by an inhomogeneous grid with a total number of cells of $300 \times 300 \times 144$. In the inner region, $1.5 A \times 1.5 A \times 0.2 A$, the cells have a constant size of $0.01 A$. In the rest of the domain, the cell size increases to a maximum in the outer boundary of the computational domain as a geometric progression with factor 1.045. The outer boundary of the disk was defined by a cylinder with radius $R_{\mathrm{ext}}=5.2 \mathrm{~A}$ behind which the distribution of gas-dynamic values remains constant and defined by the initial conditions.

The initial conditions were adopted in the following manner: (1) the disk mass, velocity, and temperature distribution as in Section 4.1 ; (2) to accelerate the simulations, we assume the presence of the gap in the central region of the disk, i.e., the inner 


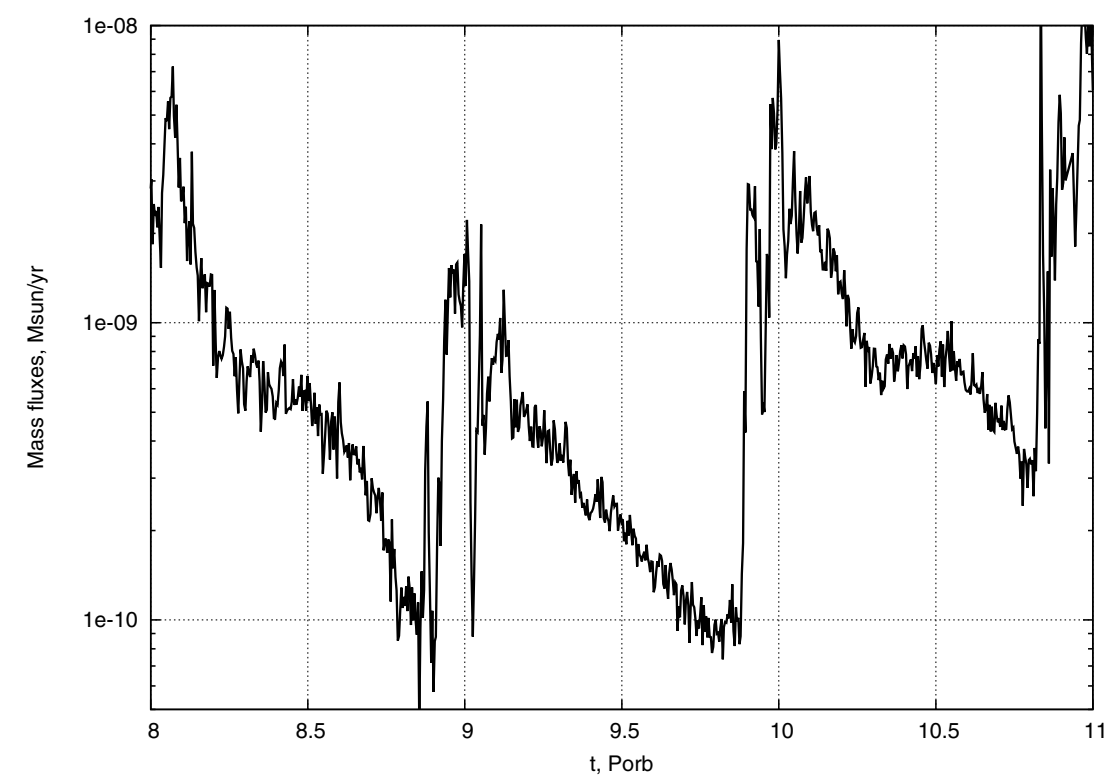

Figure 12. Accretion rate on the primary. Note that accretion outbursts occur at periastron passage.

radius of the circumbinary disk was equal to $0.3 \mathrm{AU}(\simeq 2 \times A)$, as derived from A1989 modeling of the infrared spectral energy distribution of AK Sco.

The simulations have been left to run for $\sim 10$ orbital periods until quasi-steady state is reached.

The inner boundary condition is set by the stars, represented by spherical surfaces of radii $2 \times R_{*} \simeq 0.014$ AU. A free inflow condition is set at the boundaries of the cells intersecting the spheres at any time. Cells released from the stellar surfaces are filled with "vacuum" values $\rho_{0}, T_{0}, \boldsymbol{v}_{0}$, i.e., by highly rarefied gas. We adopted $\rho_{0} \approx 10^{-20} \mathrm{~g} \mathrm{~cm}^{-3}, T_{0} \approx 10^{2} \mathrm{~K}$, and $v_{0}=0$ for these cells.

\subsection{Modeling Results}

The structure of the gas flow obtained from the simulations consists of a circumbinary disk, a gap, circumstellar accretion disks, and a system of shock waves and tangential discontinuities. These elements are outlined in Figure 10. The spiral shock waves (bow shocks) formed due to the supersonic motion of the components of the binary system channel the gas within the gap into two flows: an outward flow sending the material back to the inner border of the disk and an inward flow that drags the gas into the stars, leading to mass accretion; these two flows are marked in Figure 10 as I and II, respectively. Close to the stars, the mass flow enters into the Roche lobe; mass can then accrete either in the primary or in the secondary, marked as III and IV in the figure, respectively. A stationary shock wave forms in the area between both stars where the flows collide.

Note that in the inner region of the circumbinary disk the velocity distribution is non-Keplerian and gas motion is governed by bow shocks and binary component gravity wells. We also found that the size and the shape of the gap are substantially determined by the bow shocks. The bow shock wave has a spiral shape, one end of the bow shock is located near the circumstellar disk, while the other end resides inside the circumbinary disk. In the flow structure this appears as if the bow shock was connecting the stellar accretion disk to the circumbinary disk with a thick spiral arm. There are two bow shocks in the system, one per star, making for a two-arm spiral.
However, those arms are not accretion streams and they should not be misinterpreted as such flow features inside which the matter flows from the circumbinary disk to the accretion disk. When the matter of the gap passes through the bow shock it forms the dense spiral behind the wave, but some part of it flows to the accretion disk and the rest of it flows back to the circumbinary disk. These two flows are separated by the head-on collision point visible in the velocity field on the forefront of the bow shock. Remarkably, these streams are not distinguishable on the density distribution as two separate streams; indeed, they look like a single thick spiral arm.

Figure 11 displays the evolution of the inner region of computational domain during a single orbital period. Twelve panels show the density distribution at different times (phases) evenly distributed over the orbital period, starting at periastron. The AK Sco binary system has a substantially elliptical orbit which results in an interesting phenomenon. When the system approaches periastron, the outer boundaries of the circumstellar disks (and the accretion streams passing by) get close enough one to each other to effectively lose the angular momentum, leading to an increase of the accretion rate by a factor of 2-3 (see Figure 12). A tangential discontinuity is produced at the point where the primary and secondary accretion flows get in contact because, at the interface between them, the matter orbiting around the primary moves in the opposite direction that the matter orbiting the secondary (see Figures 11 and 13). Collisional heating, development of instabilities (like Rayleigh-Taylor instability on the interface), and shock wave formation will efficiently remove the angular momentum and, henceforth, lead to an accretion outburst.

The structure of the gas flow in the orbital plane of the system at periastron and apastron is shown in more detail in Figures 13 and 14, respectively. The velocity field presented in Figures 13 and 14 shows the direction of all gas flows in the system. The velocity distribution along with shock waves (shown in these figures as density enhancements) gives a full description of the flow structure in the system. At periastron passage, a complex pattern of shock waves appears between the components of the binary system. Since the accretion rate significantly increases at periastron, this results in an increase of the high-energy radiation 


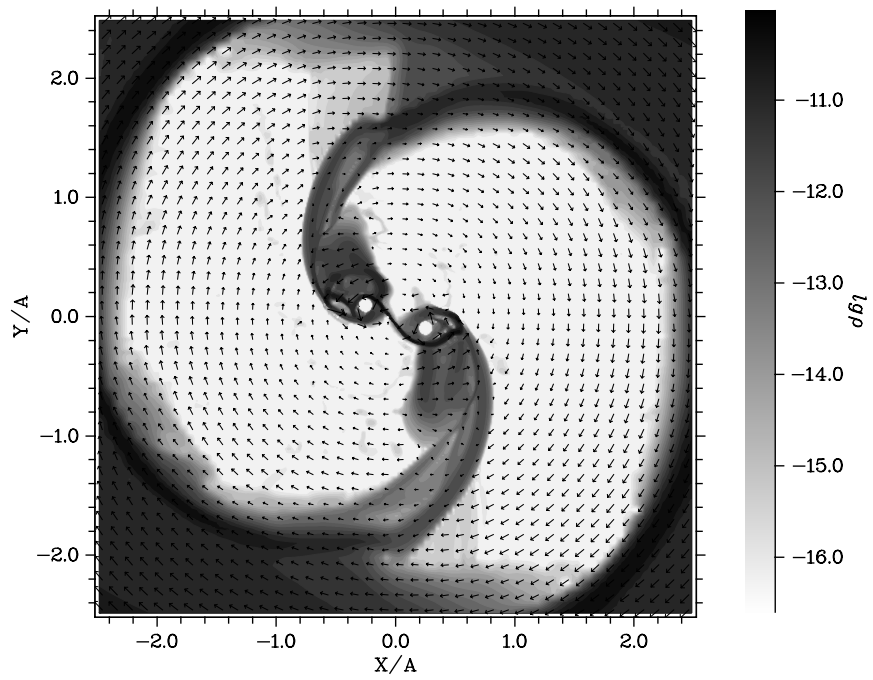

Figure 13. Density and velocity distribution in the orbital plane of the binary system at periastron passage. The density is displayed in the laboratory reference frame, but velocity vectors are translated to the rotating stellar frame to assist the interpretation.

flux. At the same time the gap becomes cleaner, i.e., contains less matter, at periastron than at apastron mainly due to the significant increasing of the accretion rate and more efficient removal of the matter from the gap, by stronger shock waves caused by faster orbital motion. As a result, the total amount of mass in the gap is seen to vary, as expected, in anti-phase with the accretion rate.

\section{A GENERAL SCENARIO FOR AK Sco}

Based on the three XMM-Newton observations that scarcely monitor the system from periastron to apastron, there seems to be an orbital modulation of the high-energy tracers of magnetospheric activity. The energy radiated in these tracers, both in the UV and X-ray, increases with the tide and the accretion rate: it is higher at periastron than at apastron. Moreover, the maximum flux is observed at phase $\phi=0.117$, when stellar occultation could occur (A1989), thus precluding any association with eclipse like phenomena.

In Figure 15, the variation of the radiative energy release during the binary cycle is shown for X-ray and UV wavelengths (from this work) in the bottom panels. At the top, data from the optical monitoring of AK Sco run in 1982 by Manset et al. (2005, hereafter MBB2005) are displayed for comparison. The variation of the $U$ and $V$ magnitudes, as well as the variation of the polarization in the $U$ band during the orbital cycle, is plotted. Note that $U$ and $V$ magnitudes remain around a basal level between periastron and phase 0.4 , after the flux increases significantly to reach the maximum close to apastron. Finally, at phase 0.65 , the flux decreases rapidly at the same time that the polarization increases. The correlation between the increase of polarization and the decrease in the optical band flux is interpreted by MBB2005 as a dusty structure crossing the line of sight.

Returning back to our observations, X-ray data clearly show a variation of the gas column during the orbit. The gas column varies from $N_{\mathrm{H}}=0.35_{-0.29}^{+0.29} \times 10^{21} \mathrm{~cm}^{-2}$ at periastron to $N_{\mathrm{H}}=1.11_{-0.46}^{+0.49} \times 10^{21} \mathrm{~cm}^{-2}$ at apastron (see Column 3 in Table 5). This variation can be translated into an $A_{V}$ variation making use of the conversion from hydrogen column density to optical extinction derived recently by Guever \& Oezel (2009)

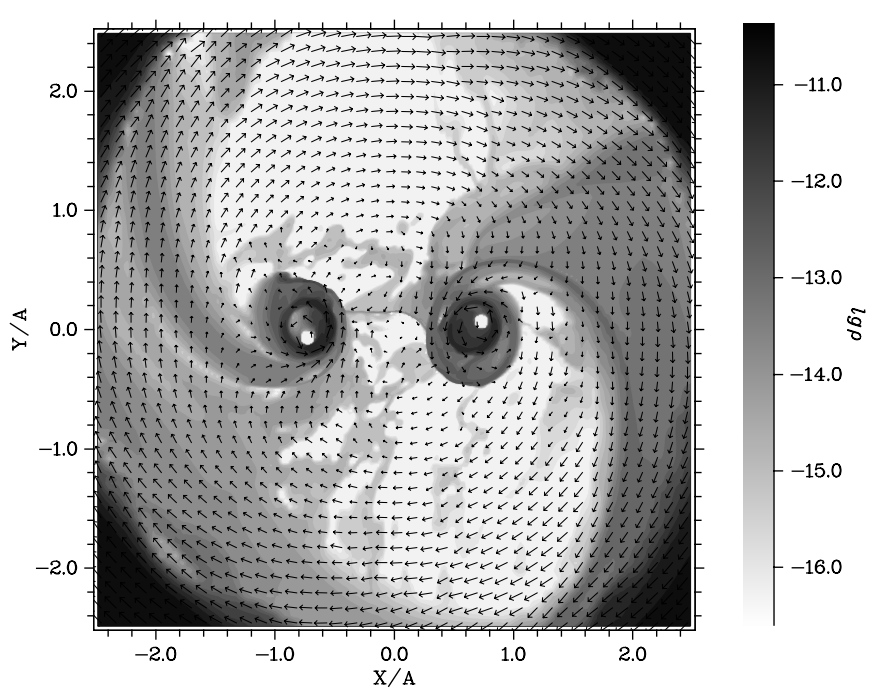

Figure 14. Same as Figure 13 at apastron passage.

from X-ray-based H I column densities in the Galaxy: $N_{\mathrm{H}}=$ $(2.21 \pm 0.09) \times 10^{21} A_{V}$ (mag). According to this conversion, $A_{V}$ would vary from $0.16 \mathrm{mag}$ at periastron to $0.50 \mathrm{mag}$ at apastron; this last value agrees with the fiducial $A_{V}$ value determined from optical observations (see Table 1). As will be shown below, this variation in gas column is consistent with the variation in polarization detected by MBB2005.

Serkowski et al. (1975) established that polarization by interstellar dust takes its maximum value at a wavelength of $\lambda_{\max } \simeq 0.45 \mu \mathrm{m}-0.8 \mu \mathrm{m}$. This maximum polarization $\left(p_{\max }\right)$ correlates with $E(B-V)$ as $p_{\max } \leqslant 9.0 E(B-V)$ (Whittet et al. 1992). Since $R=4.3=A_{V} / E(B-V)$ in AK Sco (MBB2005), this expression can be transformed into $p_{\max } \leqslant$ $2.1 A_{V}=0.95 N_{\mathrm{H}, 21} \mathrm{~cm}^{-2}$, with $N_{\mathrm{H}, 21}=N_{\mathrm{H}} / 10^{21}$ making use again of the Guever \& Oezel (2009) conversion. According to this scaling, a variation in $N_{\mathrm{H}}$ as the derived from the $X M M$ / EPIC observations would correspond to a variation in $p_{\max }$ of $0.7 \%$, in good agreement with the polarization measurements (see Figure 15). Note that for the large sample of stars studied by Serkowski et al. (1975), the median value is $\left\langle\lambda_{\max }\right\rangle=0.55 \mu \mathrm{m}$ which roughly corresponds to the $V$ band and agrees well with MBB2005.

In summary, the maximum gas column detected in the XMM-Newton observations in 2011 is comparable to the maximum gas/dust column reported by MBB2005 from a photometric/polarimetric optical monitoring of the system in 1982. This suggests that the absorption could be produced by a rather stable pattern, such as warp waves in the disk, instead of tracing a casual passage of a dust cloud by the line of sight. Yet, further observations are required to confirm this point.

The variable extinction affects significantly the UV light curve obtained with OM. A variation, $\Delta A_{V}$, of $0.34 \mathrm{mag}$ from periastron to apastron corresponds to an increment in $\Delta A_{\mathrm{UV}}$ in the OM band of $0.82 \mathrm{mag}$. This implies that while the $\mathrm{X}$-ray flux would be enhanced at periastron, the UV flux in the OM- $U V M 2$ window would be maximum at apastron. This, apparently puzzling, behavior is consistent with the detection of an enhancement of the O I flux (see Section 3.3). Note that the UV flux detected by OM comes from two main sources.

1. The direct flux from the stellar magnetospheres and the accretion shocks that it is expected to be maximum at 


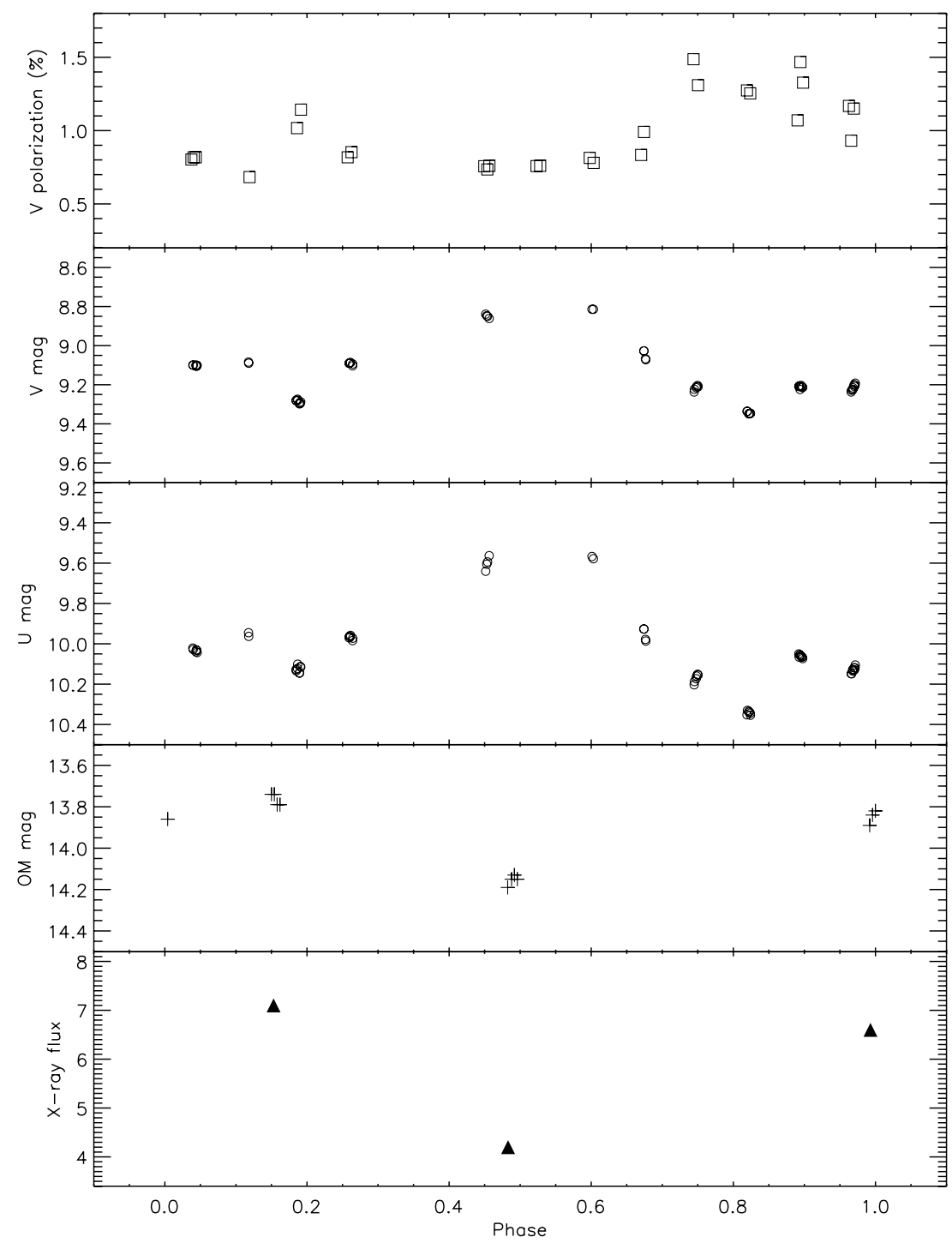

Figure 15. Summary of AK Sco variability during the orbital cycle. Optical polarization ( $V$ band) and $U$ and $V$ magnitudes are plotted in the three upper panels using data from MBB2005. UV and X-ray variations are plotted from data in this work.

periastron since the accretion rate (and the X-ray flux) is maximum at this phase (see also Figure 12).

2. The high-energy (X-ray, extreme and far-UV) stellar flux reprocessed in the circumstellar environment and diffused into the line of sight.

Since the OM- $U V M 2$ flux is higher at apastron than at periastron, reprocessed radiation must be the dominant contributor to the UV flux in this band. This is consistent with the results from the simulations shown in Figure 11. The variable gravitational field induced by the binary in the disk produces a variable distribution of circumstellar material; it acts like a gravitational piston that drags material from the inner border of the disk into the gap. At periastron, there is an accretion outburst and the gap becomes rather clean; thus, though the direct UV flux is higher, the contribution from the reprocessed UV radiation is minimum. At apastron, however, the gap is filled with gas and the stars are closer to the inner border of the circumbinary disk than at periastron. Henceforth, the reprocessing of the highenergy radiation from the star into lower energies, as well as the diffusion of this radiation into the line of sight, is more efficient. This provides a general scenario to interpret AK Sco data that it is also consistent with previous results in the optical range; note that as the variation in the optical bands is also dominated by reprocessed radiation (the Balmer continuum in the $U$ band and the emission lines in the $V$ band), the flux should also be maximum at apastron in these bands.

\section{CONCLUSIONS}

This work has allowed us to define a working scenario to explain the main properties of AK Sco. This star has revealed itself to be a unique new type of interacting binary system. We have shown that AK Sco eccentric orbit acts like a gravitational piston. At apastron, matter is efficiently dragged from the inner disk border, filling the inner gap and producing accretion streams that end as ring-like structures around each component of the system. At periastron, the ring-like structures get in contact, leading to angular momentum loss, and thus producing an accretion outburst. Additional relevant conclusions from this work are as follows.

1. In spite of its peculiarities, AK Sco satisfies the same UV flux-flux relations as the rest of the TTSs. Thus, the strong tidal wave, driven by the close and highly eccentric orbit, 
does not seem to modify the distribution of the energy released among the various UV spectral tracers.

2. AK Sco X-ray luminosity is two orders of magnitude smaller than the one observed in the TTSs. It is unclear whether this is mimicking the well-known drop of the magnetic activity around F8 types in main-sequence stars or whether it could be associated with other physical processes since not all UV bright and accreting TTSs are detected in X-ray.

3. The modest monitoring run with XMM-Newton suggests that accretion is enhanced at periastron in anti-phase with the filling of the inner gap of the disk, and reprocessing of high-energy radiation into the UV recombination bands. This behavior agrees with the predictions from numerical simulations.

This work has been partly funded by the Ministry of Science and Innovation of Spain through grant AYA2008-06423-C0301/03. A.S. and D.B. were partly supported by RFBR and Russian Federation President grants.

Facilities: XMM (OM), XMM (EPIC)

\section{REFERENCES}

Albacete Colombo, J. F., Flaccomio, E., Micela, G., Sciortino, S., \& Damiani, F. 2007, A\&A, 464, 211

Alencar, S. H. P., Melo, C. H. F., Dullemond, C. P., et al. 2003, A\&A, 409, 1037 (A2003)

Andersen, J., Lindgren, H., Hazen, M. L., et al. 1989, A\&A, 219, 142 (A1989)

Arnaud, K. 1996, in ASP Conf. Ser. 101, XSPEC: The First Ten Years, ed. G. H. Jacoby \& J. Barnes (San Francisco, CA: ASP), 17

Arnaud, K. 2004, BAAS, 36, 934

Artymowicz, P., \& Lubow, S. H. 1994, ApJ, 421, 651

Ayres, T. R., Fleming, T. A., Simon, T., et al. 1995, ApJS, 96, 223

Bate, M. R., \& Bonnel, I. A. 1997, MNRAS, 285, 33

Bisikalo, D. V., Boyarchuk, A. A., Kuznetsov, O. A., \& Chechetkin, V. M. 2000, ARep, 44, 26

Bonito, R., Orlando, S., Peres, G., Favata, F., \& Rosner, R. 2007, A\&A, 462,645

Bowen, I. S. 1947, PASP, 59, 196

Boyarchuk, A. A., Bisikalo, D. V., Kuznetsov, O. A., \& Chechetkin, V. M. 2002, Mass Transfer in Close Binary Stars (London: Taylor and Frances)

Calvet, N., Basri, G., Imhoff, C. L., \& Giampapa, M. S. 1985, ApJ, 293, 575

Carkner, L., Feigelson, E. D., Koyama, K., Montmerle, T., \& Reid, I. N. 1996, ApJ, 464, 286

Fateeva, A. M., Bisikalo, D. V., Kaygorodov, P. V., \& Sytov, A. Y. 2011, Ap\&SS, 335,125

Favata, F., Fridlund, C. V. M., Micela, G., Sciortino, S., \& Kaas, A. A. 2002, A\&A, 386, 204

Fireman, G. F., \& Imhoff, C. L. 1989, NASA IUE Newsletter, 40, 10

Giampapa, M. S., Calvet, N., Imhoff, C. L., \& Kuhi, L. V. 1981, ApJ, 251, 113

Gómez de Castro, A. I. 2009a, Ap\&SS, 320, 97

Gómez de Castro, A. I. 2009b, ApJL, 698, L108
Gómez de Castro, A. I., \& Franqueira, M. 1997, International Ultraviolet Explorer-Uniform Low Dispersion Archive, Access Guide, No. 8 (Noordwijk: ESA)

Gómez de Castro, A. I., \& Lamzin, S. A. 1999, MNRAS, 304, L41

Gómez de Castro, A. I., López-Santiago, J., \& Talavera, A. 2013, MNRAS, 429, L1

Gómez de Castro, A. I., \& Marcos-Arenal, P. 2012, ApJ, 749, 190 (GdCMA2012)

Gómez de Castro, A. I., \& Verdugo, E. 2001, ApJ, 548, 976

Gómez de Castro, A. I., \& von Rekowski, B. 2011, MNRAS, 411, 849

Guedel, M., Padgett, D., Audard, M., et al. 2007, BAAS, 38, 753

Guedel, M., Skinner, S. L., Audard, M., Briggs, K. R., \& Cabrit, S. 2008, A\&A, 478,797

Guenther, R., \& Kley, W. 2002, A\&A, 387, 550

Günther, H. M., Schmitt, J. H. M. M., Robrade, J., \& Liefke, C. 2007, A\&A, 466, 1111

Guever, T., \& Oezel, F. 2009, MNRAS, 400, 2050

Gullbring, E., Calvet, N., Muzerolle, J., \& Hartmann, L. 2000, ApJ, 544, 927

Hamilton, C. M., Johns-Krull, C. M., Mundt, R., Herbst, W., \& Winn, J. N. 2012, ApJ, 751, 147

Hanawa, T., Ochi, Y., \& Ando, K. 2010, ApJ, 708, 485

Hartmann, L. 2009, in Protostellar Jets in Context, ed. K. Tsinganos, T. Ray, \& M. Stute (Astrophysics and Space Science Proceedings Series; Berlin: Springer), 23

Herbst, W., \& Moran, E. C. 2005, ApJ, 630, 400

Herczeg, G. J., Linsky, J. L., Johns-Krull, C. M., \& Wood, B. E. 2002, ApJ, 572,310

Holm, A., \& Rice, G. 1981, NASA IUE Newsletter, 15, 74

Ingleby, L., Calvet, N., Bergin, E., et al. 2011, ApJ, 743, 102

Jensen, E. L. N., \& Mathieu, R. D. 1997, AJ, 114, 301

Johns-Krull, C. 2007, ApJ, 664, 975

Kaigorodov, P. V., Bisikalo, D. V., Fateeva, A. M., \& Sytov, A. Y. 2010, ARep, 54,1078

Lamzin, S. A. 1998, ARep, 42, 322L

Linsky, J. L., Bornmann, P. L., Carpenter, K. G., et al. 1982, ApJ, 260, 670

Manset, N., Bastien, P., \& Bertout, C. 2005, AJ, 129, 480 (MBB2005)

Mathieu, R. D., Stassun, K., Basri, G., et al. 1997, AJ, 113, 1841

Morrison, R., \& McCammon, D. 1983, ApJ, 270, 119

Pérez, M. R. 1991, ESA IUE Newsletter, 38, 27

Serkowski, K., Mathewson, D. S., \& Ford, V. L. 1975, ApJ, 196, 261

Shi, J.-M., Krolik, J., Lubow, S. H., et al. 2012, ApJ, 749, 118

Smith, R. K., Brickhouse, N. S., Liedahl, D. A., \& Raymond, J. C. 2001a, APJL, 556, L91

Smith, R. K., Brickhouse, N. S., Liedahl, D. A., \& Raymond, J. C. 2001b, in ASP Conf. Ser. 247, Spectroscopic Challenges of Photoionized Plasmas (San Francisco, CA: ASP), 161

Stickland, D. J. 1980, ESA IUE Newsletter, 5, 30

Sytov, A. Y., Kaigorodov, P. V., Fateeva, A. M., \& Bisikalo, D. V. 2011, ARep, 55,793

Talavera, A. 2011, Technical Report XMM-SOC-CAL-TN-0019 issue 6.0, XMM-Newton Optical and UV monitor (OM) Calibration Status, ESA; http://xmm2.esac.esa.int/docs/documents/CAL-TN-0019.pdf

de Val-Borro, M., Gahm, G. F., Stempels, H. C., \& Pepliński, A. 2011, MNRAS, 413, 2679

Valencic, L. A., Clayton, G. C., \& Gordon, K. D. 2004, ApJ, 616, 912

van Leeuwen, F. 2007, A\&A, 474, 653

von Rekowski, B., \& Brandenburg, A. 2004, A\&A, 420, 17

Whittet, D. C. B., Martin, P. G., Hough, J. H., et al. 1992, ApJ, 386, 562 